\title{
Nucleation of Super-Critical Carbon Dioxide in a Venturi Nozzle
}

\author{
D. Jarrahbashi, S. R. Pidaparti, D. Ranjan
}

\begin{abstract}
Pressure reduction at the entrance of the compressor in supercritical $\mathrm{CO}_{2}$ Brayton cycles may cause nucleation and create a mixture of vapor and liquid droplets due to operation near the saturation conditions. Transient behavior of the flow after nucleation may cause serious issues in operation of the cycle and degrade the materials used in the design. The nucleation behavior of supercritical carbon-dioxide inside a venturi nozzle near the critical point is computationally studied. A transient compressible 3D Navier-Stokes solver, coupled with continuity, and energy equations have been implemented. In order to expedite the simulations, Fluid property Interpolation Tables (FIT) based on a piecewise biquintic spline interpolation of Helmholtz energy have been integrated with OpenFOAM to model $\mathrm{S}_{-} \mathrm{CO}_{2}$ properties. The mass fraction of vapor created in the venturi nozzle has been calculated using homogeneous equilibrium model (HEM). Nucleation behavior has been shown to be very sensitive to the inlet pressure, inlet temperature, and flow rate. The flow conditions that led to nucleation were identified. Nucleation was observed in the throat area and divergent section of the nozzle for mass flow rates from $0.050 \mathrm{Kg} / \mathrm{s}$ to $0.065 \mathrm{~kg} / \mathrm{s}$, inlet pressure from 7.8 to 7.4 MPa for fixed exit pressure equal to 7.28 MPa. The inception of high-vapor-content nucleation was first observed in the throat area away from the side walls that remained confined to the throat region in later times. However, near the walls, a high liquid-content two-phase region was detected, first in the divergent section. At later times, the two-phase region was convected downstream toward the nozzle exit. Nucleation in central plane was associated with larger pressure drop and higher vapor-content (higher volume fraction); whereas lower pressure drop and more liquid-content (lower volume fraction) was observed near the walls.
\end{abstract}




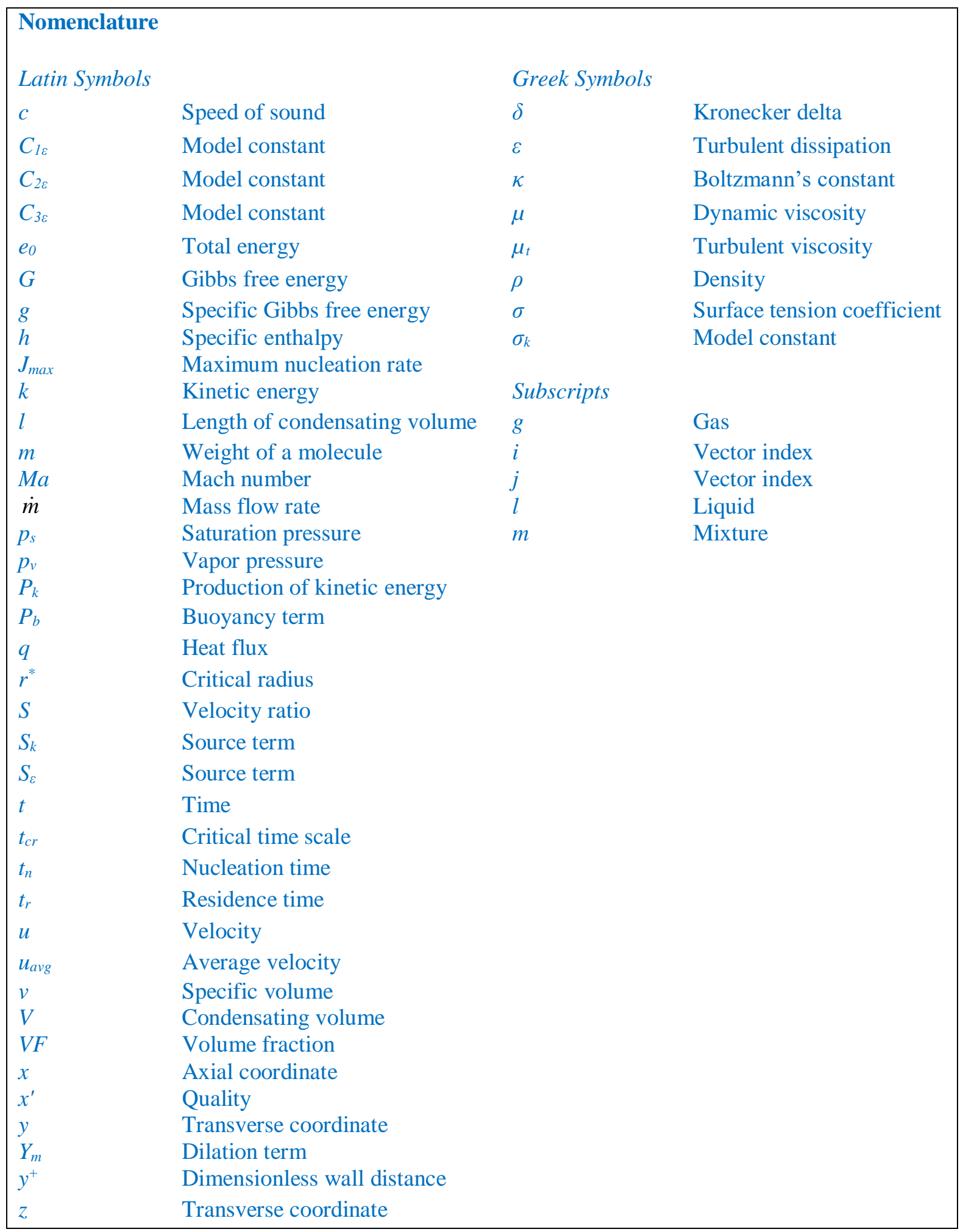




\section{Introduction}

Supercritical carbon dioxide Brayton cycles offer a number of benefits over competing steam Rankine cycles, namely reducing the size of turbomachinery, increased efficiency, reduction of greenhouse gases, and reduced capital cost $[1,2]$. The augmented heat transfers and low specific volume of supercritical $\mathrm{CO}_{2}$ $\left(\mathrm{S}-\mathrm{CO}_{2}\right)$ cycles make them a good candidate to replace traditional steam Rankine cycles in future [3, 4]. As the specific volume decreases near the critical point $\left(7.38 \mathrm{MPa}, 31^{\circ} \mathrm{C}\right)$, the compressor work decreases and thermal efficiency increases. So, the size of the heat exchangers and turbomachinery substantially reduces compared to water or helium Brayton cycles $[5,6]$. Despite the recent progress in academic level, more research is required to advance the research and manufacturing of $\mathrm{S}-\mathrm{CO}_{2}$ power cycles for commercial purposes [6]. Power cycles for variety of heat sources, e.g. nuclear, in sodium fast Reactor (SFR) and fluoride high temperature reactor (FHR), solar energy, and fossil fired power plants [7] can benefit from $\mathrm{SCO}_{2}$ as a working fluid.

There is a pressing need to understand the transition of the working fluid $\mathrm{S}-\mathrm{CO}_{2}$ near the critical point. Resolution of issues with cavitation and fluid instabilities operating near and in the two-phase region is of great importance. It is expected that at some degree of pressure reduction where the pressure and temperature are within the vapor dome, nucleation, i.e. either cavitation or condensation or both occur. In rotating machinery this can take place at the end of the turbine blades and at the entrance to the compressor causing loss of power and instability in compressor performance [8]. This is of serious concern with respect to operation and the materials used in designing these systems. Materials erosion, primarily at high temperatures, is an important issue particularly because the expected life times of these components have to be 20 years or more.

The sensitivity of the nucleation behavior to the compressor inlet conditions near the critical point has been demonstrated in Figure 1. We developed a one-dimensional isentropic flow model for a nozzle with inlet to throat area equal to 17 to understand the behavior of the flow by inspecting the phase-change 
diagram. In this model, by assuming choked flow conditions in the throat region, the inlet pressure has been fixed at 7.40 MPa and the inlet temperature varies from supercritical to subcritical conditions.

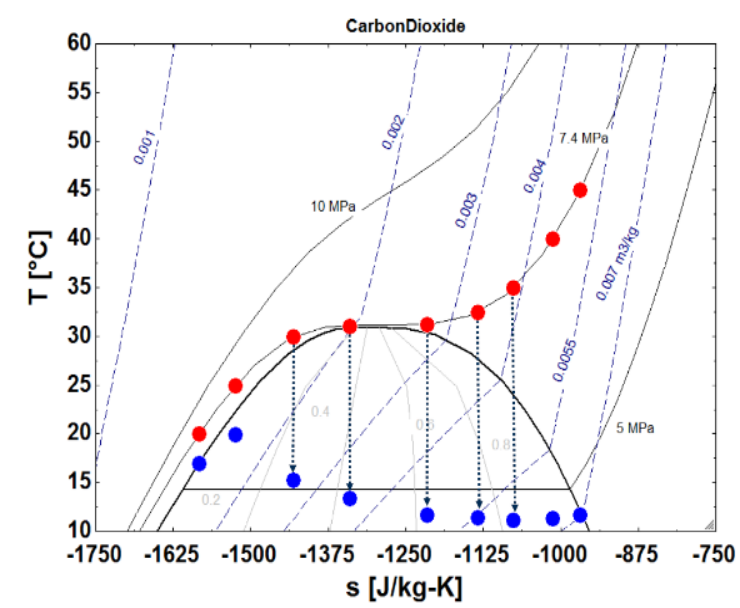

(a)

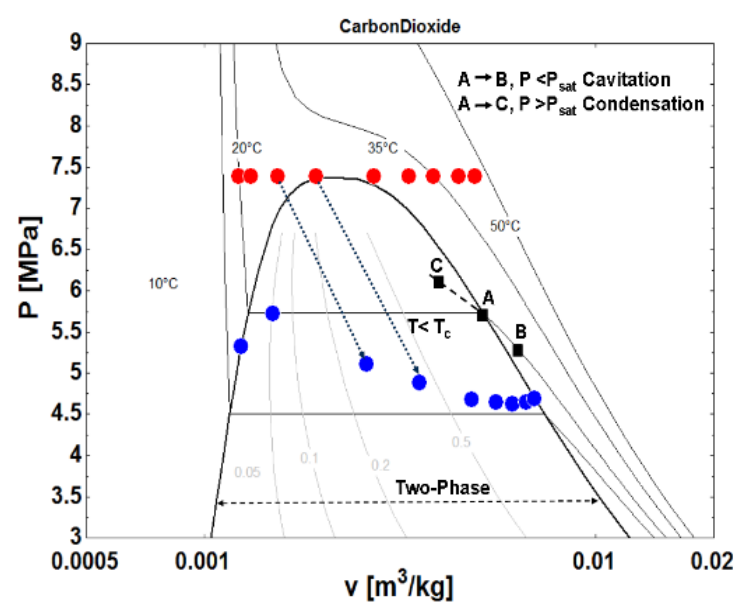

(b)

Figure 1. Predictions of $1 D$ isentropic model; entering the two-phase region with inlet conditions fixed at 7.40 MPa and subcritical and supercritical temperature on (a) $T-s$, (b) $P-v$ diagrams. (The red and blue symbols show the inlet and throat conditions, respectively.)

Red symbols in Figure 1 (a) represent the $\mathrm{CO}_{2}$ stagnation conditions at the venturi nozzle inlet. The blue symbols along the constant entropy line below each red symbol show the corresponding choked flow conditions at the throat. It can be deduced from the $T$-s diagram shown in Figure 1 (a) that the nucleation process is extremely sensitive to the inlet stagnation conditions. To the right side of the critical point and approaching the saturated vapor line, i.e. the metastable region, all the inlet stagnation conditions lead to a two-phase mixture with higher vapor volume fraction, i.e. smaller volume fractions of condensate droplets at the throat. The opposite behavior is expected approaching the saturated liquid line from the critical conditions toward subcritical inlet temperatures, i.e. to the left of the critical point.

Near the critical pressure, a small variation in inlet temperature compared to the critical temperature may alter the nucleation behavior from condensation to cavitation as evident from the $T$-s and $P-v$ diagrams. 
The nucleation behavior can be assessed by comparing the local pressure to the saturation pressure at the local temperature. This situation has been demonstrated in Figure 1 (b), where $A$ is located on the saturation vapor line and points $B$ and $C$ are on the same isotherm. In case the pressure drops below the saturation pressure at the local temperature, i.e. moving from point $A$ to $B$, cavitation is expected. On the same isotherm line, by moving from $A$ to $C$, the pressure exceeds the saturation pressure and condensation is expected. Distinguishing between these two limits of nucleation and its sensitivity to the inlet conditions near the critical point is significant in designing compressors for supercritical conditions. Cavitation phenomenon is expected to be more detrimental in degrading the compressor blades since the cavitation bubbles burst in a very short time scales near the surface whereas condensation may cause instability in the compressor and energy loss rather than serious erosion. Further experiments are required to identify the effects of cavitation/ condensation on compressors.

It is well documented that the numerical simulations near the critical point can be challenging $[8,9]$. Sharp variation of thermodynamic properties near the critical point and their strong deviation from the ideal gas law affects the stability of the numerical simulations/ schemes and highlights the significance of using real gas properties in simulations. The numerical study of $\mathrm{CO}_{2}$ flow in the main axial compressor in the neighborhood of the critical point by Takagi [9] demonstrated that the solution did not converge for a few stages of the compressor using real gas properties. Thus, they used modified ideal gas properties to obtain convergence in all stages of the compressor. Therefore, there is a substantial need in performing stable numerical simulations with real gas thermodynamic properties near the critical point and in the two-phase regions. There is lack of knowledge in predicting the transient phase change behavior of $\mathrm{S}-\mathrm{CO}_{2}$ near the critical point.

Nucleation of $\mathrm{S}-\mathrm{CO}_{2}$ in supersonic flows in converging-diverging nozzles has been experimentally studied by measuring the static pressure drops within the nozzle for the ejectors used in refrigeration cycles [10]. In an ejector, the converging-diverging nozzle gets choked and the pressure of the fluid converts into momentum to energize lower-momentum suction flow. The nucleation process in these nozzles may cause shock and expansion waves since the local speed of sound will change when $\mathrm{S}-\mathrm{CO}_{2}$ enters the two-phase 
region. Computational studies based on real-fluid properties of $\mathrm{CO}_{2}$ and local mass and energy transfer between phases have been performed and validated against experiments away from the critical point [11]. However, there are very few experimental and numerical studies of subsonic $\mathrm{S}-\mathrm{CO}_{2}$ in the converging diverging nozzle near the critical point applicable to the compressor inlet conditions in $\mathrm{S}-\mathrm{CO}_{2}$ Brayton cycles. Numerical simulations and experiments of $\mathrm{S}-\mathrm{CO}_{2}$ flow in a converging diverging nozzle performed by Lettieri [12] indicated the significance of two-phase effects on the turbomachinery design. A nondimensional criterion, i.e. the ratio of the nucleation time to residence time was defined to determine the inception of nucleation. Nucleation time is the time required for stable liquid droplets to form which depends on the rate of the expansion of the flow whereas the residence time represents the local flow time scale under saturated conditions. In a recent numerical study, this criterion was applied to the candidate centrifugal $\mathrm{S}-\mathrm{CO}_{2}$ compressor $[12,13]$. They predicted that condensation was unlikely in the radial compressors away from the critical point in the $\mathrm{S}-\mathrm{CO}_{2}$ power cycles since the nucleation time was much longer than the flow residence time. However, very close to the critical point, the residence time increased and transition from single-phase to two-phase became more probable. The purpose of this research work is to study the nucleation process of $\mathrm{S}-\mathrm{CO}_{2}$ by simulating the transient flow in a venturi nozzle using real gas properties. In this study, the nucleation process of $\mathrm{S}-\mathrm{CO}_{2}$ in the immediate vicinity of the critical point has been investigated by solving transient 3D Navier-Stokes and energy equations by implementing the real properties of $\mathrm{S}-\mathrm{CO}_{2}$ into the computational code. The proposed framework of the nucleation process of S$\mathrm{CO}_{2}$ in the nozzle serves as a foundation for the definition of the impact of two-phase flows in turbomachinery operating in the vicinity of the critical point. The computational results provide useful information for designing experimental set-up with optical access. For flow visualization, the density gradients on the nozzle walls play a significant role in capturing the flow physics. Therefore, detecting the condensating and cavitating regions inside the nozzle and on the walls via simulations is very helpful to design appropriate optical diagnostics to observe and quantify the number density of the droplets and bubbles in the two-phase regions. 


\section{Computational Model \& Governing Equations}

The flow of $\mathrm{S}-\mathrm{CO}_{2}$ in a converging-diverging nozzle with inlet temperature and pressure close to the critical point has been studied. The nozzle has been designed to provide the required pressure drop to initiate the phase change process in the throat area. A schematic of the converging-diverging nozzle used in these simulations has been shown in Figure 2. The nozzle has a rectangular $3 D$ profile with a $3 \mathrm{~mm}$ constant depth in the $z$ direction perpendicular to the paper. The observations from the phase change process due to local pressure changes as a result of acceleration or deceleration of the flow when surface area changes within the nozzle have been presented. Based on homogenous mixture assumption, the two phases are considered as one combined mixture of liquid/ vapor in these simulations. The properties of the mixture, i.e. mixture pressure, mixture velocity, saturation temperature at the mixture pressure, and volume fraction have been found from the conservation of mass, momentum, energy, and equation of state.
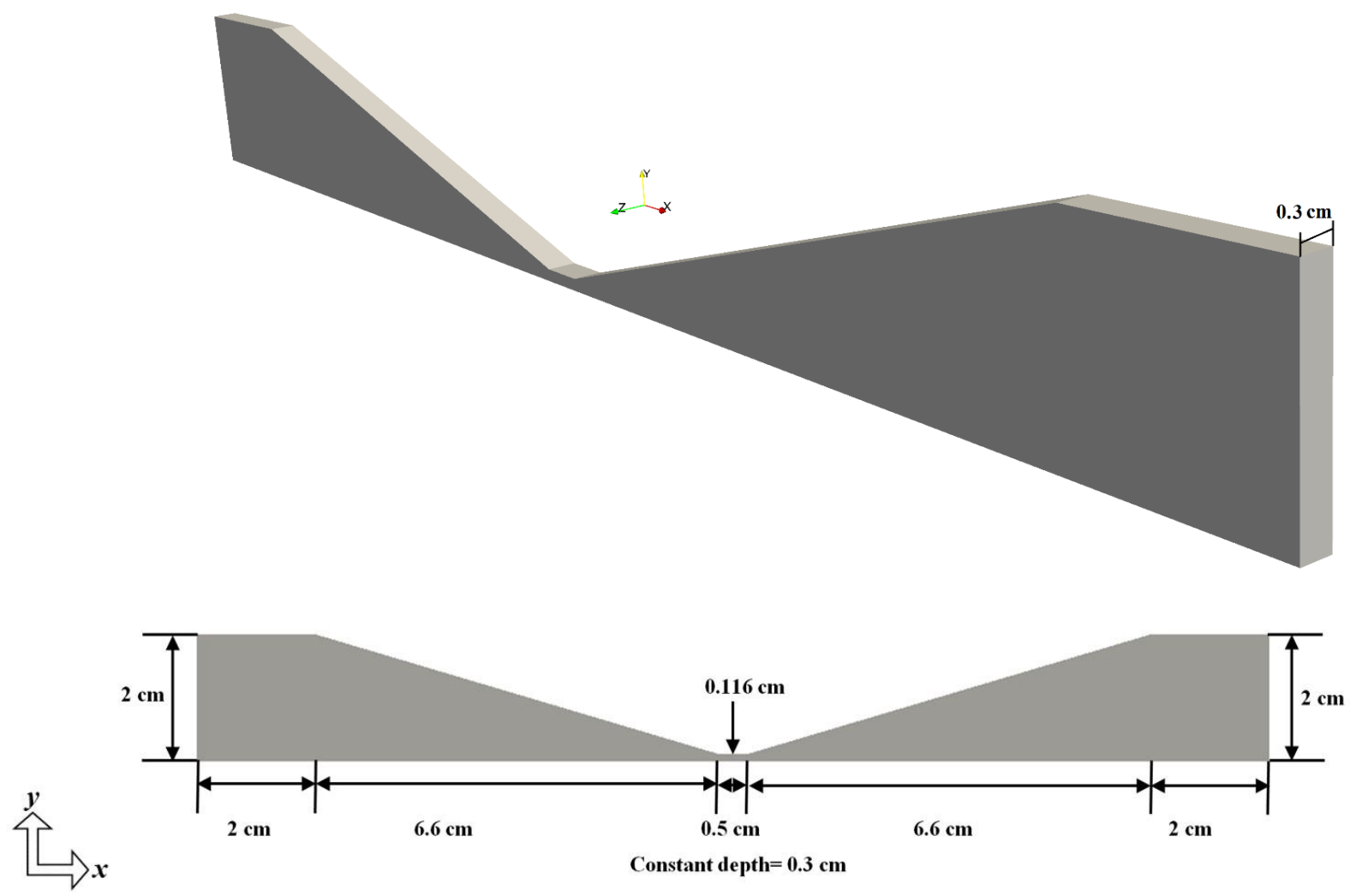

Figure 2. Schematic of the converging-diverging nozzle with rectangular profile with constant depth of 3

$\mathrm{mm}$. 
The density and energy of each discrete phase have been obtained from the equation of state and the mixture density as: $\rho_{m}=V F \rho_{g}+(1-V F) \rho_{l}$ and $e_{m}=V F \rho_{g} e_{g}+(1-V F) \rho_{l} e_{l}$, where $\rho_{m}, e_{m}$, and $V F$ are the mixture density, mixture energy, and volume fraction and subscripts $l$ and $g$ refer to liquid and gas phases, respectively. The mass fraction of liquid and vapor created due to nucleation are sought. By interpreting the mixture quantities, the same set of compressible Navier-Stokes and energy equations as for a single-phase flow have been solved. Thus, the velocity has been obtained from a single momentum equation for the mixture. Continuity equation has been solved for each phase to calculate the volume fraction. A closure to these equations is provided by modeling the properties of $\mathrm{CO}_{2}$. FIT (Fluid property Interpolation Tables), an algorithm which utilizes a modified version of biquintic spline interpolation of Helmholtz free energy and all of its derivatives, have been implemented in OpenFOAM [14] to accurately model the properties of $\mathrm{CO}_{2}$ required to solve the governing equations near the critical point. The underlying property data of FIT is obtained from REFPROP (Software for fluid properties developed by NIST). However, FIT generates fluid properties much faster than REFPROP within acceptable errors [15].

The governing equations are as follows:

Conservation of mass: $\quad \frac{\partial \rho_{m}}{\partial t}+\frac{\partial}{\partial x_{j}}\left(\rho_{m} u_{m_{j}}\right)=0$

Conservation of momentum: $\frac{\partial \rho_{m} u_{m_{i}}}{\partial t}+\frac{\partial}{\partial x_{j}}\left[\rho_{m} u_{m_{i}} u_{m_{j}}+P \delta_{i j}-\tau_{i j}\right]=0$

Conservation of Energy: $\quad \frac{\partial \rho_{m} e_{0 m}}{\partial t}+\frac{\partial}{\partial x_{j}}\left[\rho_{m} e_{0 m} u_{m_{j}}+u_{m_{j}} P+q_{j}-u_{m_{j}} \tau_{i j}\right]=0$

where $u_{m}, p, \tau_{i j}, \delta_{i j}, e_{0 m}$, and $q$ are mixture velocity, pressure, viscous stress, kronecker delta, total mixture, total mixture energy, and heat flux, respectively and $e_{0 m}=e_{m}+\frac{u_{m i} u_{m i}}{2}$. The standard $k-\varepsilon$ turbulence model with enhanced wall treatment has been chosen. Transport equations for standard $k-\varepsilon$ model are as follows: 


$$
\begin{aligned}
& \frac{\partial \rho_{m} k}{\partial t}+\frac{\partial}{\partial x_{j}}\left(\rho_{m} k u_{m_{j}}\right)=\frac{\partial}{\partial x_{j}}\left[\left(\mu+\frac{\mu_{t}}{\sigma_{k}}\right) \frac{\partial k}{\partial x_{j}}\right]+P_{k}+P_{b}-\rho \varepsilon-Y_{M}+S_{k} \\
& \frac{\partial \rho_{m} \varepsilon}{\partial t}+\frac{\partial}{\partial x_{j}}\left(\rho_{m} \varepsilon u_{m_{j}}\right)=\frac{\partial}{\partial x_{j}}\left[\left(\mu+\frac{\mu_{t}}{\sigma_{\varepsilon}}\right) \frac{\partial \varepsilon}{\partial x_{j}}\right]+\frac{C_{1 \varepsilon} \varepsilon}{k}\left(P_{k}+C_{3 \varepsilon} P_{b}\right)-\frac{C_{2 \varepsilon} \rho \varepsilon^{2}}{k}+S_{\varepsilon}
\end{aligned}
$$

where $\mu_{t}$ is turbulent viscosity and $S_{k}, S_{\varepsilon}$ are source terms. $\sigma_{k}, \sigma_{\varepsilon}, C_{\mu}, C_{1 \varepsilon}, C_{2 \varepsilon}$, and $C_{3 \varepsilon}$ are model constants. $P_{k}$ and $P_{\varepsilon}$ are production of kinetic energy and buoyancy term, respectively. $Y_{M}$ is the contribution of the fluctuating dilation in compressible turbulence to the overall dissipation rate. Based on the equilibrium assumption, an equilibrium speed of sound has been defined to calculate the Mach number for both the single-phase and two-phase regions within the flow. The current simulation code has been validated against experimental data for studying the $\mathrm{S}-\mathrm{CO}_{2}$ flow in see-through labyrinth seals [15]. As $\mathrm{S}-\mathrm{CO}_{2}$ flows through a labyrinth seal, two-phase behavior is expected similar to the situation in the converging-diverging nozzle. The numerical model predicted the experimental results obtained for labyrinth seals very closely for various tested designs and conditions [15].

Typically, in subsonic compressible flow simulations, exit pressure and inlet mass flow rate are fixed. he inlet pressure is calculated based on these conditions assuming zero gradient conditions for pressure at the inlet. Same boundary conditions have been applied here. For velocity boundary conditions, zero gradient at the outlet and no-slip boundary conditions on all the walls have been applied. Finally, zero-gradient boundary conditions for temperature have been applied at both inlet and outlet. Isentropic $1 D$ model for the nozzle dimensions shown in Figure 2, has been used to predict the mass flow rate and exit pressure required for transition from single-phase to two-phase at the throat while the inlet condition is at supercritical pressure and supercritical temperature very close to the critical point. The boundary conditions for the simulated cased have been outlined in Table 1. For higher mass flow rate simulations, the exit pressure is fixed that allows for higher inlet pressure and higher velocity compared to lower mass flow rates. The grid independency tests were performed for medium, coarse, and fine mesh corresponding to 5.28e $3,2.64 \mathrm{e} 3$, and 1.05 e 6 cells, respectively. The maximum percent error in velocity and pressure within the nozzle with 
respect to the medium mesh was equal to $0.9 \%$ and $3 \%$ for the coarse and fine mesh, respectively. So, the medium mesh was chosen for the entire simulations here. The percent error in predicating the volume fraction and quality with respect to the medium mesh was less than $0.02 \%$ for both the coarse and fine meshes. The size of the cells near the walls satisfied the requirement of dimensionless wall distance $y^{+} \sim 1$.

\begin{tabular}{|c|c|c|c|c|}
\hline \multicolumn{5}{|c|}{ Table 1. Inlet and outlet conditions used in simulations. } \\
\hline $\begin{array}{c}\text { Mass Flow rate } \\
(\mathrm{kg} / \mathrm{s})\end{array}$ & $\begin{array}{c}\text { Inlet Pressure } \\
(\mathrm{MPa})\end{array}$ & $\begin{array}{c}\text { Exit Pressure } \\
(\mathrm{MPa})\end{array}$ & $\begin{array}{c}\text { Inlet Velocity } \\
(\mathrm{m} / \mathrm{s})\end{array}$ & $\begin{array}{c}\text { Inlet Temperature } \\
(\mathrm{K})\end{array}$ \\
\hline 0.050 & 7.62 & 7.28 & 2.71 & 305.15 \\
\hline 0.052 & 7.68 & 7.28 & 2.82 & 306.78 \\
\hline 0.065 & 7.73 & 7.28 & 3.32 & 307.22 \\
\hline
\end{tabular}

\section{Homogenous Equilibrium Model}

Homogeneous equilibrium model (HEM) has been used in this work to predict the flow behavior in the two-phase region. HEM model assumes that the velocities of both phases are equal and thermal equilibrium exists between phases. Neglecting the initial velocity, fluid velocity at any section is simply determined based on an energy balance. In this model, the mass, momentum, and energy is transferred between phases fast enough that equilibrium is reached. HEM is valid when the two phases are sufficiently well mixed; disperse particle sizes are sufficiently small to eliminate any significant relative motion between the two phases. The HEM model is valid if the gas-to-liquid velocity ratio is close to one. It should be noted that physically, there is no discontinuity between the two phases since there is a boundary layer for each phase on either side of the interface. Thus, velocity ratio is an indicator of the relative motion between the two phases. Numerous analytical and empirical correlations exist for the velocity ratio [16]. The simplest definitions in the literature have been defined as Zivi's kinetic energy model for annular flow: $S=\frac{u_{g}}{u_{l}}=\left(\frac{\rho_{l}}{\rho_{g}}\right)^{\frac{1}{3}}$ [17] and $S=\frac{u_{g}}{u_{l}}=\left(\frac{\rho_{l}}{\rho_{g}}\right)^{\frac{1}{2}}$ [18], where the former minimizes the specific two-phase mixture kinetic energy and the latter minimizes the specific two-phase momentum based on the momentum flux model. The volume fraction can be defined as: 


$$
V F=\frac{1}{1+\left(\frac{1-x^{\prime}}{x^{\prime}}\right) \frac{\rho_{g}}{\rho_{l}} \cdot S}
$$

where $x^{\prime}$ is the quality. For homogenous equilibrium model, the velocity ratio $S$ is set to one. The density difference between liquid and gas phases in $\mathrm{CO}_{2}$ near its critical pressure is much smaller than that of the water for the same range of pressure, e.g. the liquid-to-gas density ratio for $\mathrm{CO}_{2}$ varies between 2 and 3.5 as the pressure varies from 7 to $6 \mathrm{MPa}$, i.e. the lowest observed pressure in the current simulations. Therefore, the velocity ratio based on Zivi's model for this condition varies between 1.25 to 1.51 . So, the HEM model is most likely valid for the existing problem and the slip velocity between the two phases will not affect the predictions. However, further validation with experimental results is required to investigate the non-equilibrium behavior of nucleation near the critical point.

\section{Results \& Discussions}

The converging-diverging nozzle in this study has been designed so that the pressure drops below critical pressure at the end of the converging section by fixing the exit pressure at $\mathrm{P}=7.28 \mathrm{MPa}$ that is well below the critical pressure (see Figure 7- 9). In this study, the volume fraction (VF) has been defined based on Eq.6. $V F$ equal to one represents single-phase supercritical fluid if the local pressure is higher than the critical pressure at that local temperature that occurs upstream of the throat region. If the local pressure is less than the critical pressure at that local temperature, $V F=1$ corresponds to saturated vapor. This situation is expected downstream of the throat and in the diverging section of the nozzle. $V F$ less than one corresponds to the existence of a two-phase fluid. $V F$ approaching zero means that the two-phase mixture contains more liquid than vapor, i.e. condensation prevails. $V F$ close to one implies more vapor than liquid exists in the mixture, i.e. cavitation dominates.

Figure 3 and 4 show the transient behavior of nucleation in $x y-2 \mathrm{D}$ plane at two different planes in the $z$-direction, i.e. near the wall, at $z=-1.5 \mathrm{~mm}$ and at the central plane at $z=0$, respectively. The contours of $V F$ indicate that cavitation initiates in the divergent section first with a higher content of vapor, i.e. $V F=$ 
0.998 at $z=-1.5 \mathrm{~mm}$. Then, the two-phase region extends upstream and more liquid forms due to condensation. However, the cavitation initiates in the throat area in the nozzle at the central plane, i.e. $z=$ 0 as the black arrows show in Figure 4 at $t=1 \mathrm{~ms}$. As Figure 3 indicates, at $t=1.6 \mathrm{~ms}$, condensation occurs at the sharp corners in the throat area as well as the divergent section and $V F$ approaches zero in those areas. However, the two-phase region remains confined to the throat area in the central plane as Figure 4 demonstrates. The nucleation in the central plane produces more vapor than liquid since $V F$ is much larger than that of the near wall at all times. Thus, liquid droplets are expected to form near the walls and vapor tend to occupy the throat area in the central plane of the nozzle. This result is critical for designing experiments and choosing the appropriate optical diagnostics since the liquid droplets on the walls might impede the optical access to the throat area.

The comparison between the pressure drop in the central plane compared to that of near the wall versus the local saturation pressure is responsible for this change of behavior in the $z$-direction as will be discussed later in this section. Figure 3 indicates that the two-phase region has been confined at nearly a constant distance downstream of the throat in the divergent section up to $t=2 \mathrm{~ms}$ after which the two-phase region moves downstream, mostly in the liquid form. At the same time, small liquid patches detach from the bulk liquid near the throat and move downstream of the throat. At $t=2.8 \mathrm{~ms}$, a small two-phase region near the throat area with $V F>0.9$ is observed and a stream of high liquid-content mixture i.e. $V F=0.25$ travels downstream and exits the nozzle in later times (not shown here). This phenomenon can be very detrimental to the rotating parts of the compressor in actual Brayton cycles when higher liquid-content fluid moves with the swirling flow and the liquid droplets impinge on the solid parts of the cycle. Figure 4 shows that the nucleation in the throat area at $z=0$ is more prominent at early times, i.e. $t<2 \mathrm{~ms}$ after which the high vapor-content two-phase region becomes very small and remains confined to the sharp corners of the throat area as the black arrows show. In conclusion, condensation is more critical than cavitation near the walls for $\mathrm{S}-\mathrm{CO}_{2}$ at near critical conditions. 


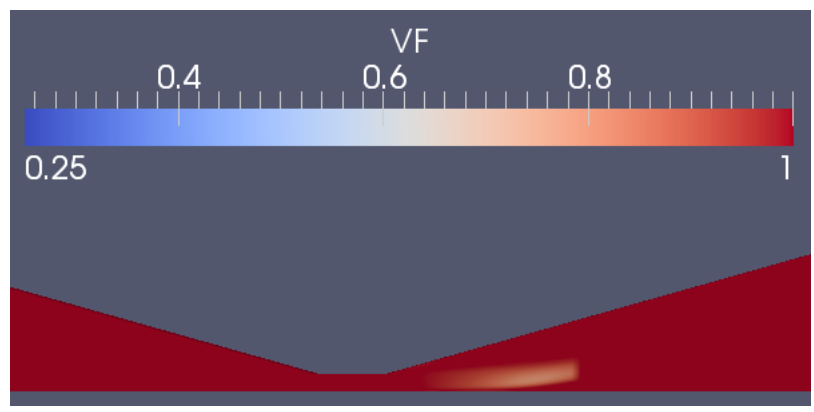

$t=1.0 \mathrm{~ms}$

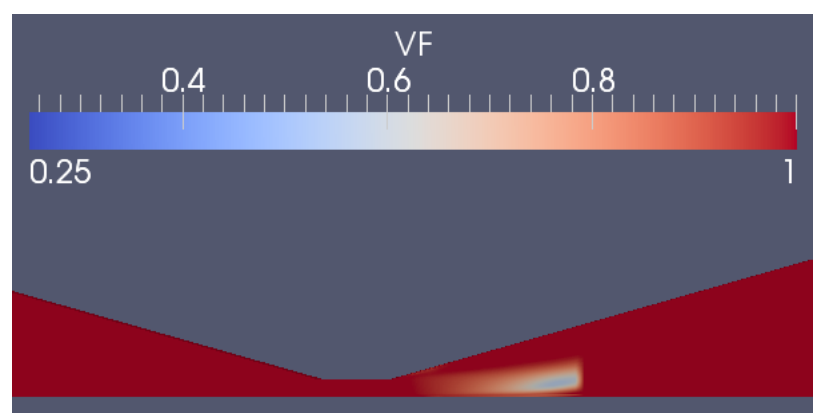

$$
t=1.2 \mathrm{~ms}
$$

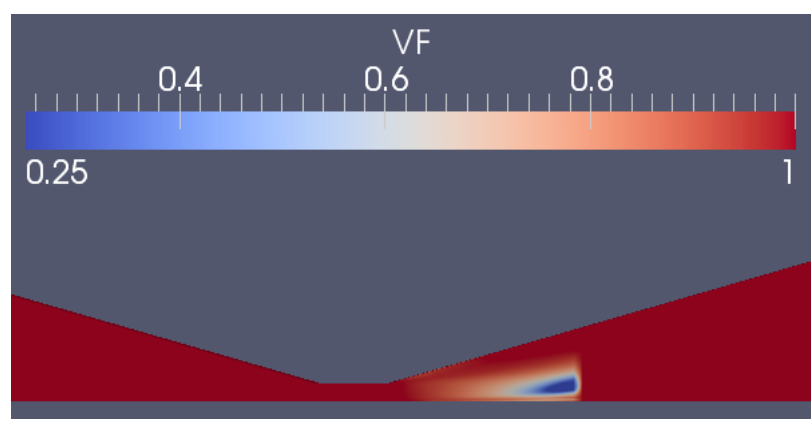

$$
t=1.4 \mathrm{~ms}
$$

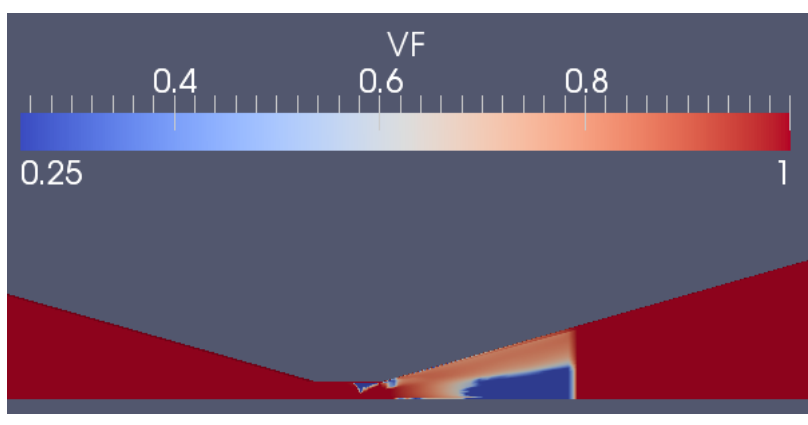

$$
t=1.6 \mathrm{~ms}
$$

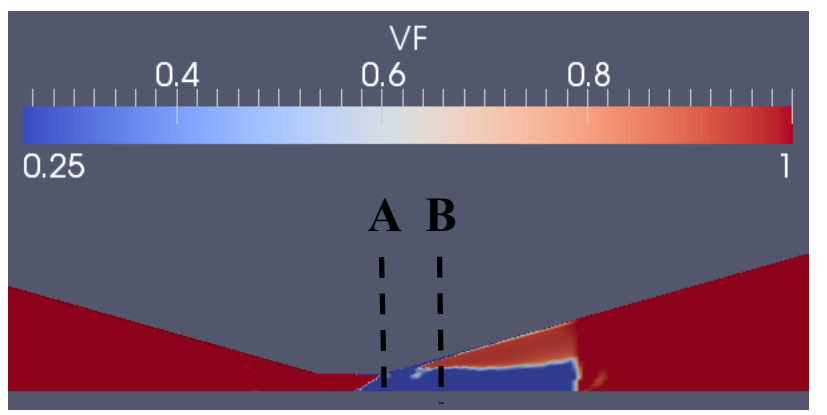

$t=2.0 \mathrm{~ms}$

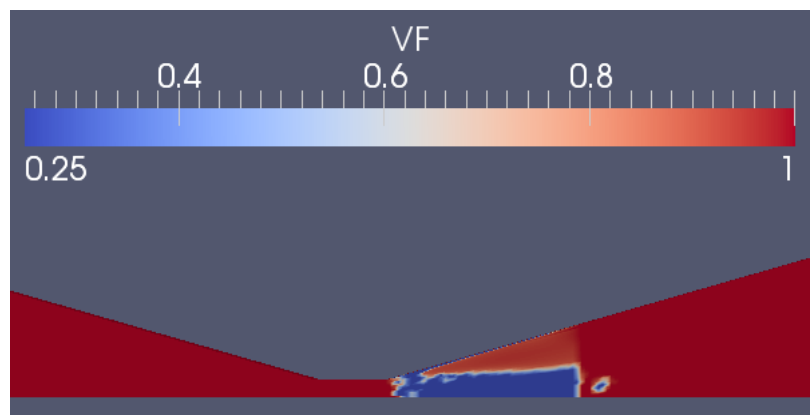

$t=2.2 \mathrm{~ms}$

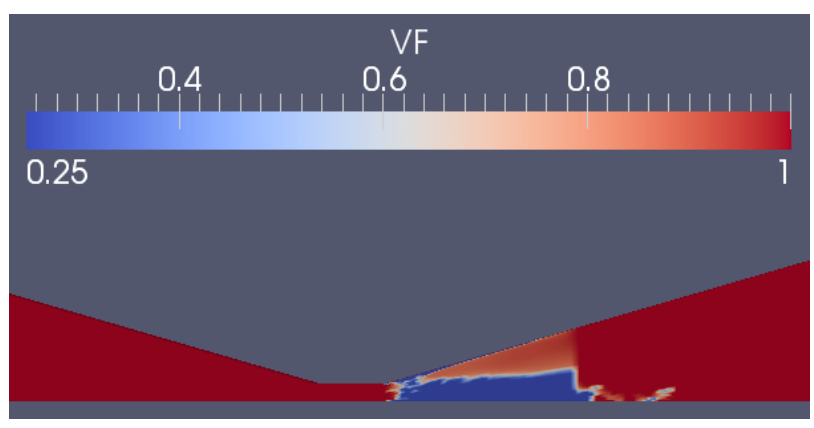

$t=2.4 \mathrm{~ms}$

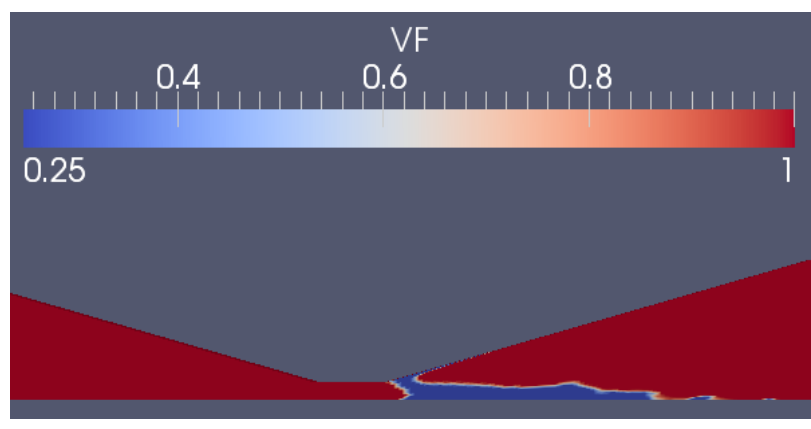

$t=2.6 \mathrm{~ms}$ 


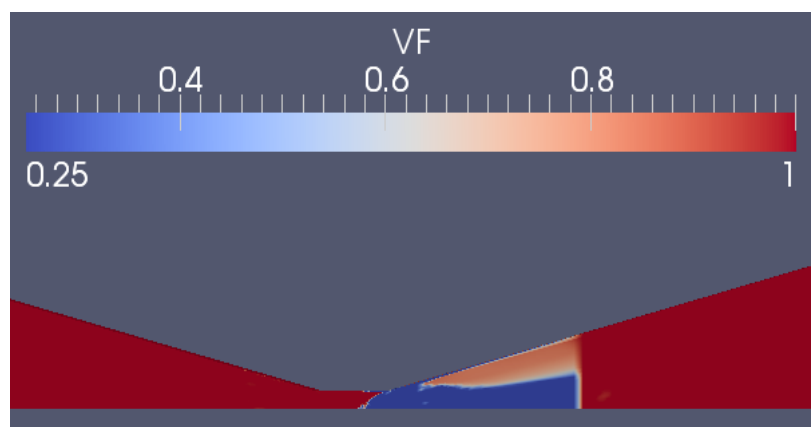

$t=1.8 \mathrm{~ms}$

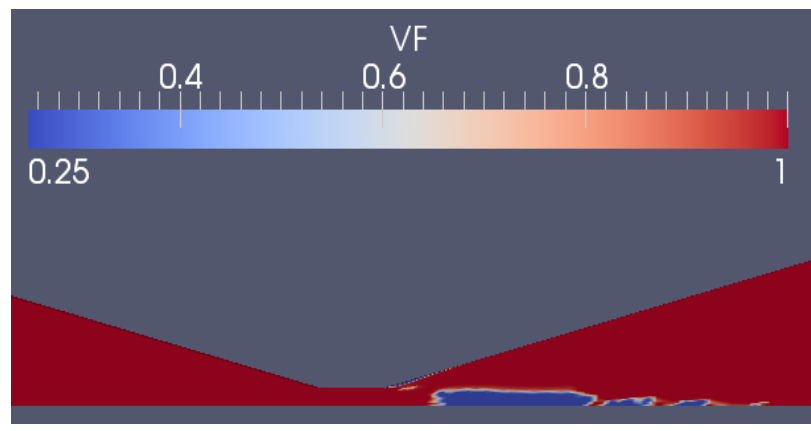

$t=2.8 \mathrm{~ms}$

Figure 3. Contours of $V F$ in the $x y$ plane indicating the transient behavior of the nucleation of $\mathrm{S}-\mathrm{CO}_{2}$ in the nozzle near the wall $(z=-1.5 \mathrm{~mm})$ for $\dot{m}=0.065 \mathrm{~kg} / \mathrm{s}$. (Flow is from left to right.)

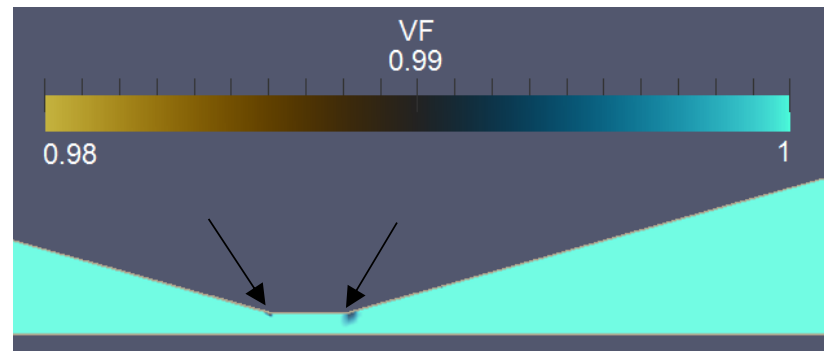

$$
t=1.0 \mathrm{~ms}
$$

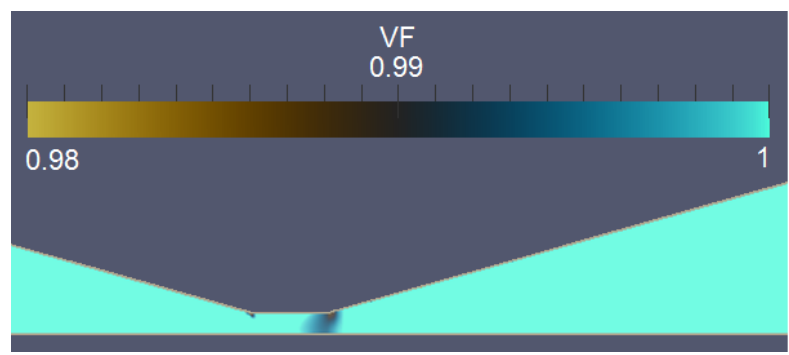

$$
t=1.2 \mathrm{~ms}
$$

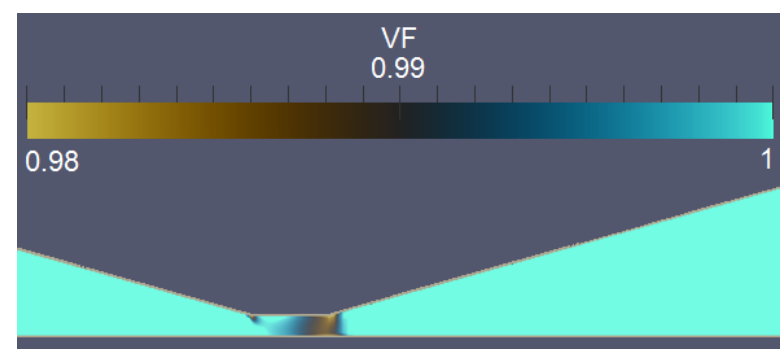

$$
t=1.4 \mathrm{~ms}
$$

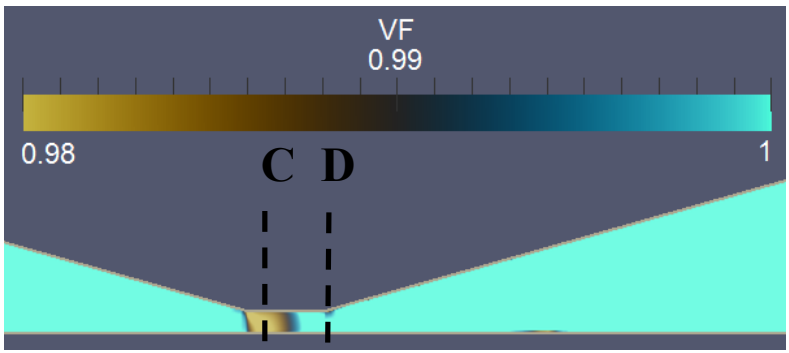

$$
t=2.0 \mathrm{~ms}
$$

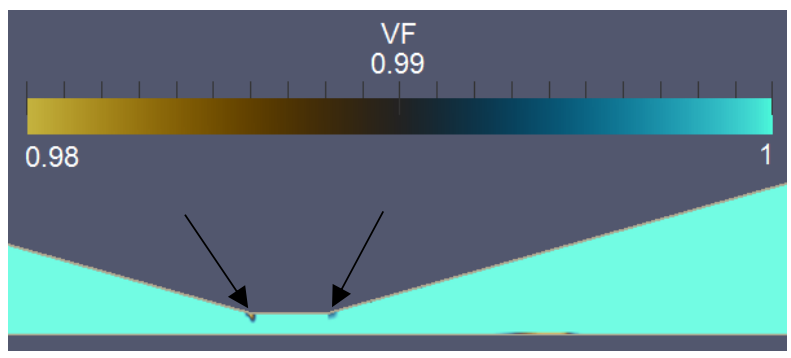

$$
t=2.2 \mathrm{~ms}
$$

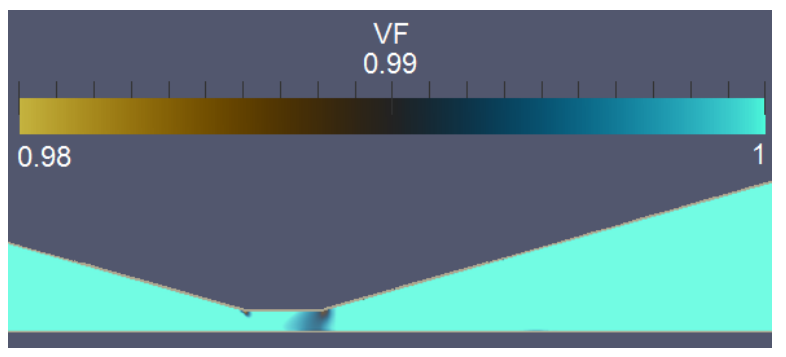

$$
t=2.4 \mathrm{~ms}
$$




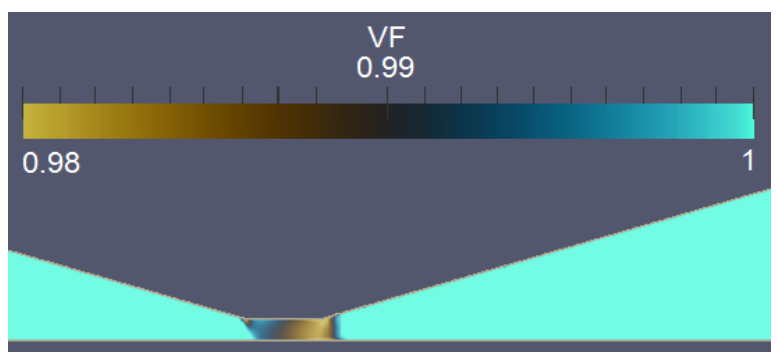

$t=1.6 \mathrm{~ms}$

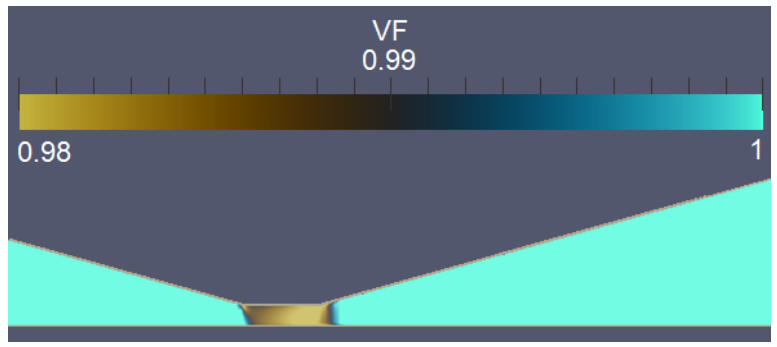

$t=1.8 \mathrm{~ms}$

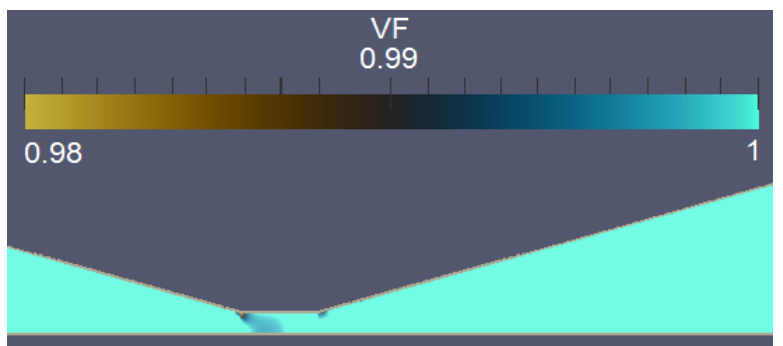

$t=2.6 \mathrm{~ms}$

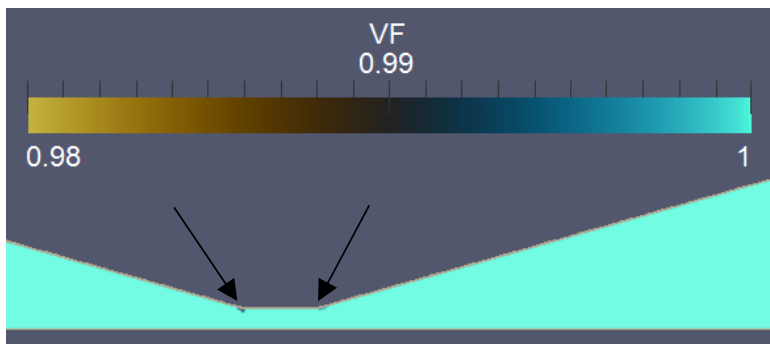

$t=2.8 \mathrm{~ms}$

Figure 4. Contours of VF in the $x y$ plane indicating the transient behavior of the nucleation of S-CO2 in the central plane $(z=0 \mathrm{~mm})$ for $\dot{m}=0.065 \mathrm{~kg} / \mathrm{s}$. (Flow is from left to right.)
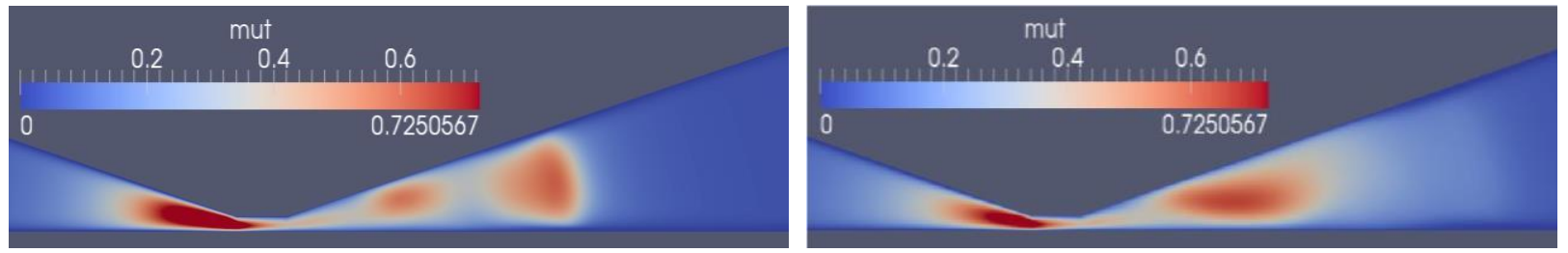

$$
t=1.4 \mathrm{~ms}
$$

$$
t=1.8 \mathrm{~ms}
$$
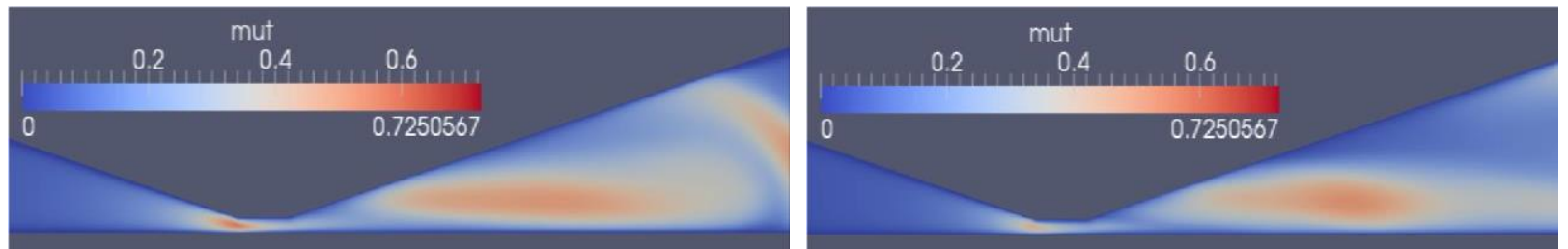

$$
t=2.2 \mathrm{~ms}
$$

$$
t=2.8 \mathrm{~ms}
$$

Figure 5. Contours of turbulent viscosity $(\mathrm{kg} / \mathrm{m} . \mathrm{s})$ in the $x y$ plane and $z=0$ indicating the development of the re-circulation region in the divergent section with time for $\dot{m}=0.065 \mathrm{~kg} / \mathrm{s}$. (Flow is from left to right.) 


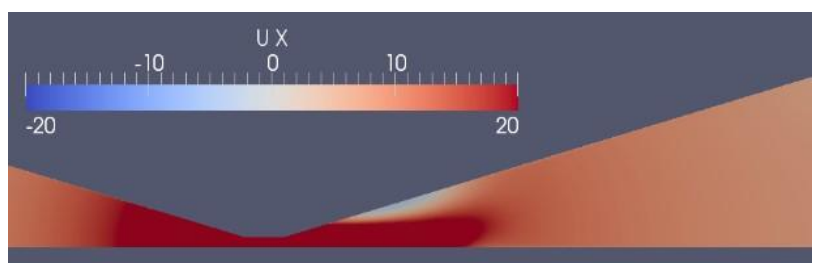

$t=1.4 \mathrm{~ms}$

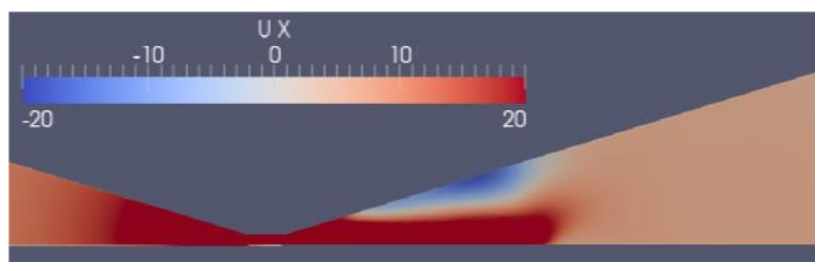

$t=2.2 \mathrm{~ms}$

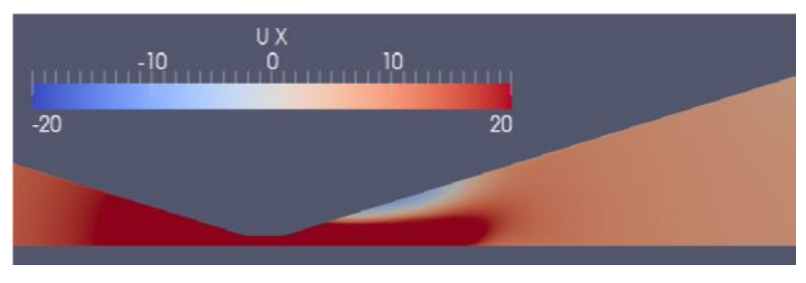

$t=1.8 \mathrm{~ms}$

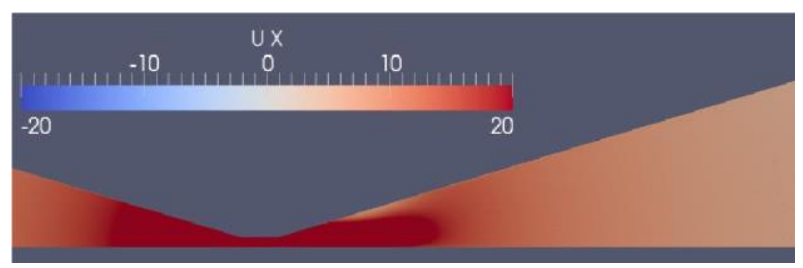

$t=2.8 \mathrm{~ms}$

(a)

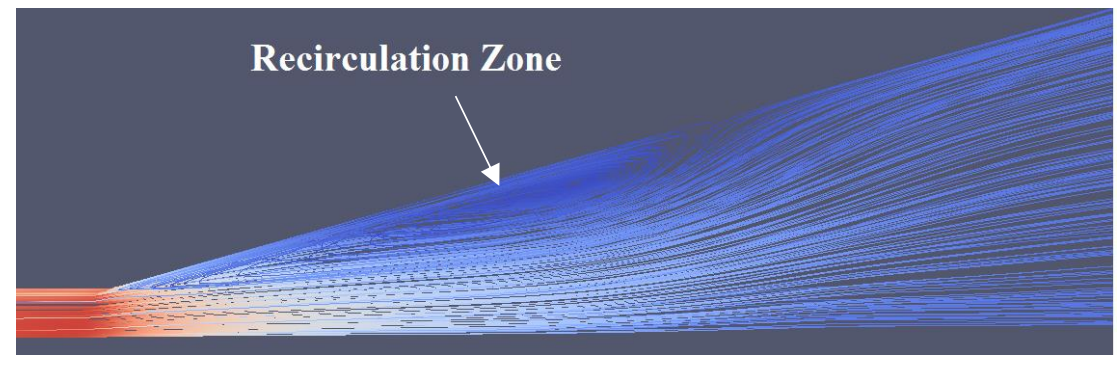

(b)

Figure 6. (a) Contours of axial velocity $(\mathrm{m} / \mathrm{s})$ and (b) streamlines in the divergent section in the $x y$-plane and $z=0$ indicating the development of the recirculation region in the divergent section with time for

$$
\dot{m}=0.065 \mathrm{~kg} / \mathrm{s} \text {. (Flow is from left to right.) }
$$

The contours of turbulent viscosity in Figure 5 demonstrate the evolution of the re-circulation area after the flow separation in the divergent section. The location of the two-phase region in the divergent section is related to the development of the flow re-circulation with time. The recirculation region in the divergent section confines the two-phase mixture in the throat region and parts of the divergent section shown in Figure 3 at $t=2.0 \mathrm{~ms}$. Later, as the re-circulation region moves downstream, the two-phase region occupies 
the divergent section and moves downstream and exit the nozzle as observed in Figure 3 at $t=2.8 \mathrm{~ms}$. The turbulent viscosity is larger near the bottom wall and smaller on the top wall in the divergent section. This explains our earlier observation that more vapor was seen near the top wall and more liquid-content mixture formed near the bottom wall. The contours of axial velocity as a function of time in Figure 6 also indicates the location of the inception and expansion of the re-circulation zone downstream of the throat, supporting the nucleation behavior observed in Figure 3. The velocity contours have been re-scaled to better portray the re-circulation region in the divergent section. Nucleation behavior on the back wall, i.e. $z=1.5 \mathrm{~mm}$, not shown here, is similar to the front wall; so, only front wall has been demonstrated here.

Figure 7 explains the different behaviors observed near the front wall and central plane in Figure 3 and 4 at $t=2 \mathrm{~ms}$, respectively. In this figure, the pressure and temperature obtained from the simulations have been averaged in the $y$-direction at different locations downstream of the venturi nozzle and plotted for both the front wall, i.e. $z=-1.5 \mathrm{~mm}$ and central plane, i.e. $z=0$. The saturated pressure at the calculated average temperature and average quality at the same downstream locations have been demonstrated in Figure 7 (a).

A schematic of the venturi nozzle has been shown at the bottom of the figure to indicate the location of interest within the nozzle. The pressure drop is larger in the convergent section and the throat area at the central plane compared to the walls since the velocity is higher near the throat and reaches minimum near the wall due to the no-slip boundary condition. At $x=0.085 \mathrm{~m}$ where the convergent section ends and throat region begins, corresponding to Section $C$ in Figure 4, the average pressure falls below the saturation pressure at that location. The corresponding value of the average $V F$ at that location is equal to 0.998 . Therefore, high-vapor content mixture is expected near the throat at the center of the nozzle. The same behavior is seen at the end of the throat region and entrance to the divergent section at $x=0.095 \mathrm{~m}$ that is associated with Section $D$ in Figure 4. At $x=0.09 \mathrm{~m}$, located in the throat region and downstream of the divergent section at $x=0.1 \mathrm{~m}$, corresponding to Section $A$ and $B$ shown in Figure 3 respectively, the obtained pressure on the front wall is larger than the value of the saturation pressure leading to more condensation on the front wall compared to the central plane. Cavitating conditions on the central plane continues in the divergent section due to the re-circulation effects near the top wall. 
Figure 7 (b) demonstrates the variation of temperature averaged along the $y$-direction with respect to the axial location in the nozzle. This figure shows that the temperature decreases from supercritical to subcritical condition with the maximum drop in the throat area and in the central plane of the nozzle.

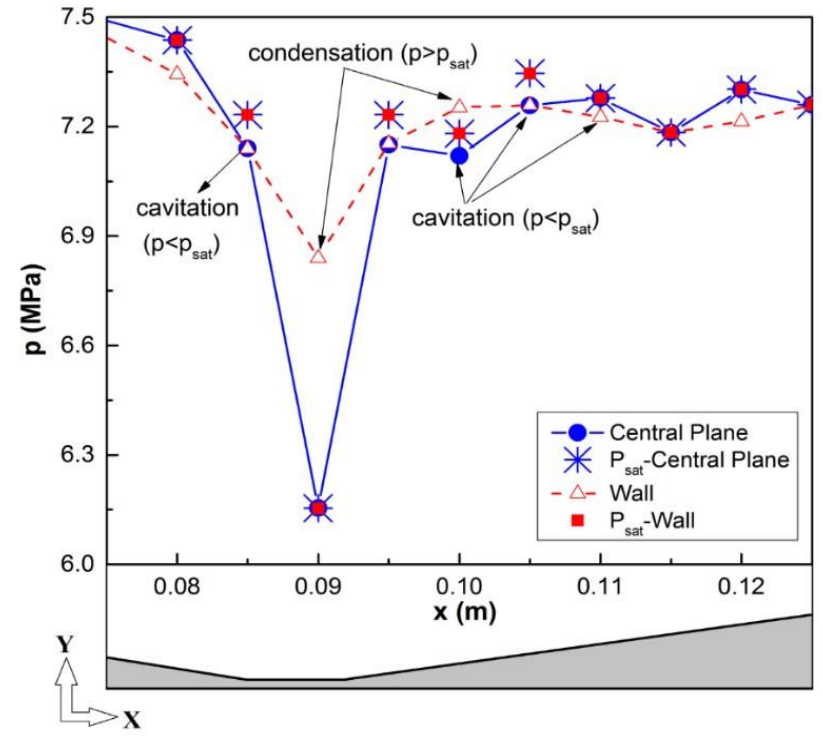

(a)

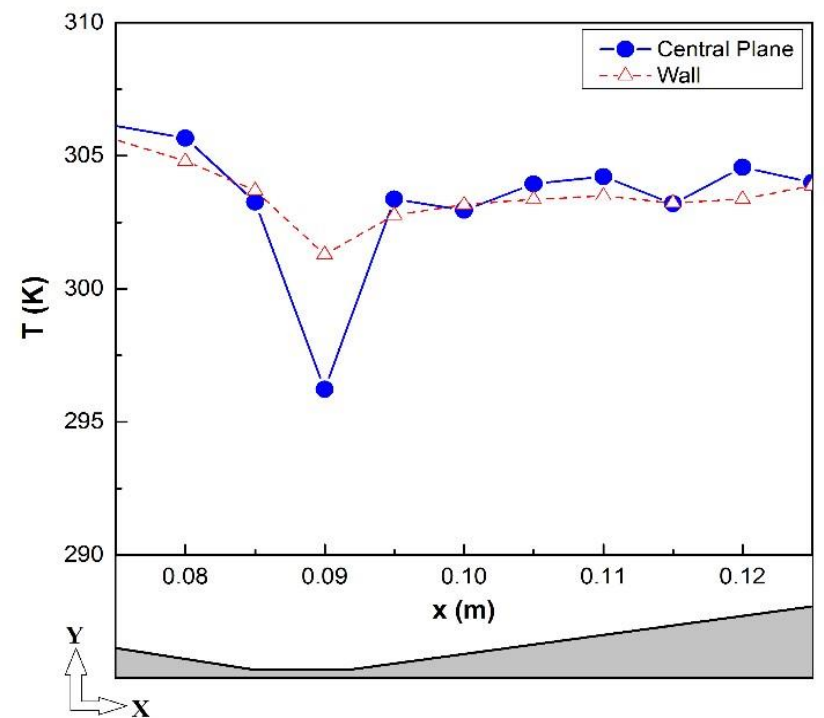

(b)

Figure 7. (a) Average pressure in $y$-direction and saturation pressure vs. $x$, (b) average temperature in $y$ direction for central plane and front wall at $t=2.0 \mathrm{~ms}$ for $\dot{m}=0.065 \mathrm{~kg} / \mathrm{s}$.

Figure 8 (a) demonstrates the contours of $V F$ on the top wall of the venturi nozzle, as the height, i.e. $y$ varies. The pressure and temperature have been shown for the same downstream locations as that of Figure 7 at $t=2.0 \mathrm{~ms}$ for $\dot{m}=0.065 \mathrm{~kg} / \mathrm{s}$. The top wall is of great interest due the formation of a relatively large re-circulation region in the divergent section and its effects on the phase change behavior. As shown in Figure 8 (a), the two-phase region is extended from the throat down to the divergent section where the recirculation region ends, after which the flow is single phase, i.e. $V F=1$ and stays as saturated vapor since the pressure remains sub-critical $(p<7.38 \mathrm{MPa})$ in the divergent section of the nozzle. As expected, condensation occurs on the two sides of the wall while cavitation takes place near the center of the nozzle 
as observed earlier. From the left to right in Figure 8 (a), in the region associated with a large density gradient in the throat area (shown as $A$ ), both liquid and vapor phases exist. A drastic drop of $V F$ is observed at the end of the throat region and entrance to the divergent section indicating large volume of liquid droplets associated with region $B$ in Figure 8 (a). Region $C$ is associated with the divergent section of the nozzle where a high-vapor content mixture prevails. Downstream of region $C$, the flow becomes single-phase saturated vapor.

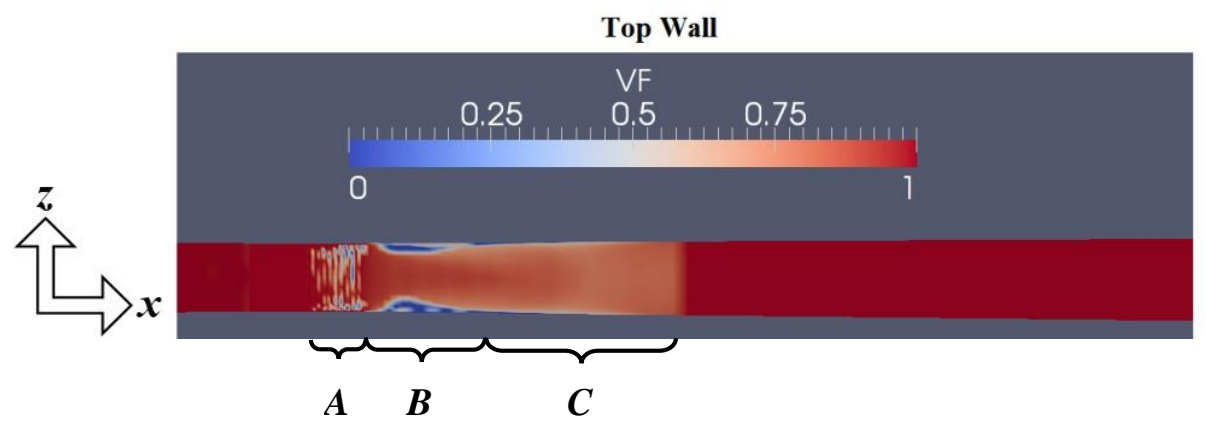

(a)

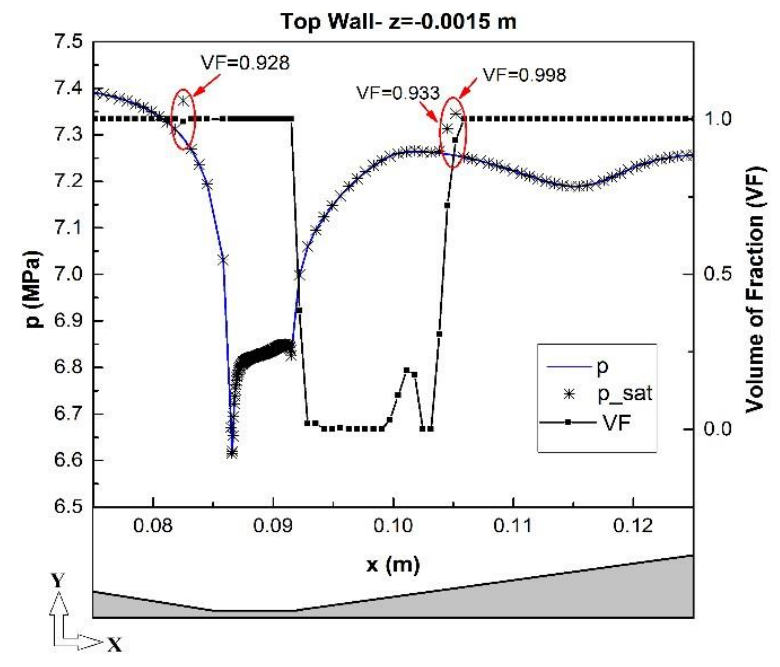

(b)

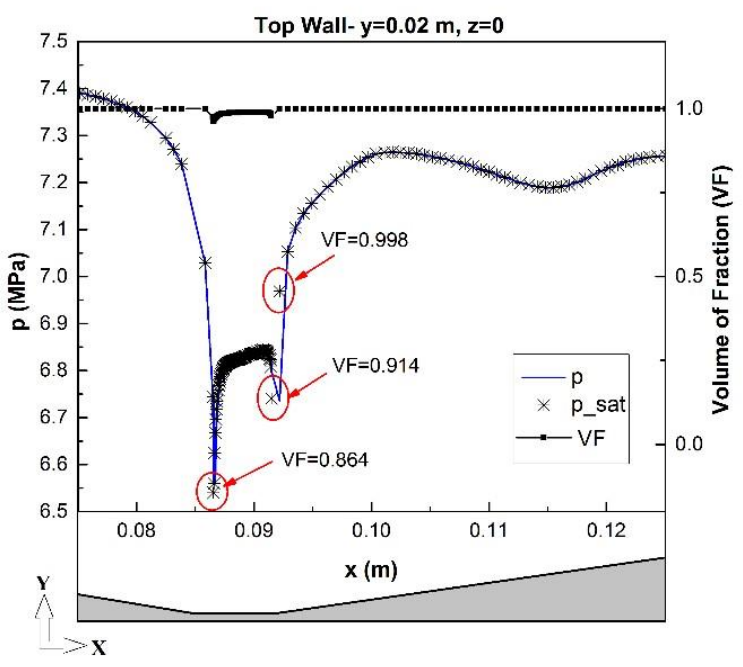

(c)

Figure 8. (a) Contours of $V F$ on the top wall at $t=2.0 \mathrm{~ms}, \dot{m}=0.065 \mathrm{~kg} / \mathrm{s}$; average pressure, saturation pressure, and $V F$ vs. downstream direction for the top wall on (b) $z=-1.5 \mathrm{~mm}$, (c) $z=0$. 
Figure 8 (b) and (c) show the pressure in the downstream location on the top wall along $z=-1.5 \mathrm{~mm}$ and $z=0$ lines, respectively. $V F$ at each downstream location is plotted on the second vertical axis on the same figure. As Figure 8 (b) indicates, $V F$ is equal to 0.928 at the center of the throat region $(x=0.085 \mathrm{~m})$. At this location, the average pressure is smaller than both the saturation pressure at the average temperature and the average quality obtained from the simulation. This region is the associated region $A$ of Figure 8 (a). As Figure 8 (c) shows, $V F$ remains larger on $z=0$ compared to $z=-1.5 \mathrm{~mm}$ in Figure 8 (b) and drops to 0.0864 and 0.914 in the throat region. Downstream of the throat area in the re-circulation region, high vaporcontent mixture persists in the center of the nozzle. The re-circulation region causes pressure variations in the downstream of the throat, i.e. $x>0.1 \mathrm{~m}$ as shown in Figure 8 (b) and (c). Drastic pressure drop is observed in the vicinity of the two sharp corners in the throat area, especially at $z=0$.

Figure 9 (a) shows the contours of $V F$ for the bottom wall, i.e. $y=0$ in the $x z$-plane at $t=2.0 \mathrm{~ms}$ for $\dot{m}$ $=0.065 \mathrm{~kg} / \mathrm{s}$. Region $A$ corresponds to the throat region where a mixture of vapor and liquid exists. Capturing the density gradients in this region might be a very challenging task experimentally. These results will help designing experiments and choosing the right optical diagnostic that can resolve the obtained density gradients in the throat region. In region $B$, more vapor forms in the middle of the channel and liquid droplets occupy the regions near the wall similar to region $B$ in Figure 8 (a). Region $C$, shows a very highliquid content mixture in contrast to Figure 8 (a) where a high-vapor content mixture formed at that region. Finally, in region $D$, a high vapor-content mixture followed by a single-phase mixture is observed. Therefore, the two-phase mixture occupies a larger volume of the nozzle downstream of the throat compared to the top wall and is associated with a higher liquid-content mixture near the bottom wall. As Figure 9 (b) and (c) demonstrate, $V F$ is smaller, i.e. higher liquid-content exists in regions $B$ and $C$ on both $z=-1.5 \mathrm{~mm}$ and $z=0$ planes compared to the top wall shown in Figure 8 (b) and (c). As expected, $V F$ is smaller on the bottom wall at $z=-1.5 \mathrm{~mm}$. The average pressure is equal to the saturation pressure at all axial locations on the bottom wall except for $x=0.12 \mathrm{~m}$ associated with region $D$ in Figure 9 (a) where a gas-liquid mixture is observed. Some fluctuations of $V F$ can be seen downstream of the throat on the bottom wall due to the flow re-circulation downstream of the throat on the top wall. As a result, liquid regions have 
been convected downstream near the wall as was shown in Figure 3. The pressure drop is less significant on the bottom wall for both $z=-1.5 \mathrm{~mm}$ and $z=0$ since the bottom wall is at a distance with respect to the sharp corners in the throat. However, the re-circulation effects are still active on the bottom wall as the pressure jumps for $x>0.1 \mathrm{~m}$ indicates.

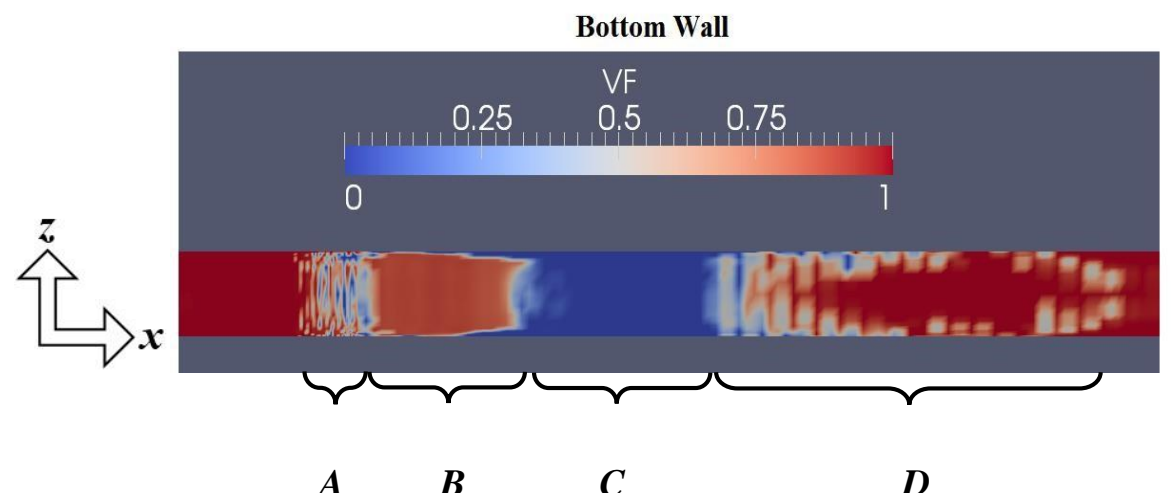

(a)

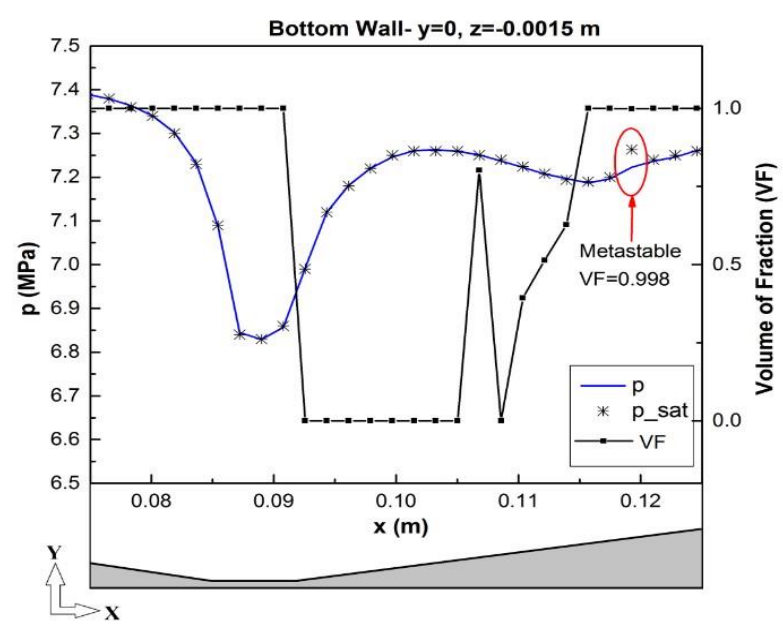

(b)

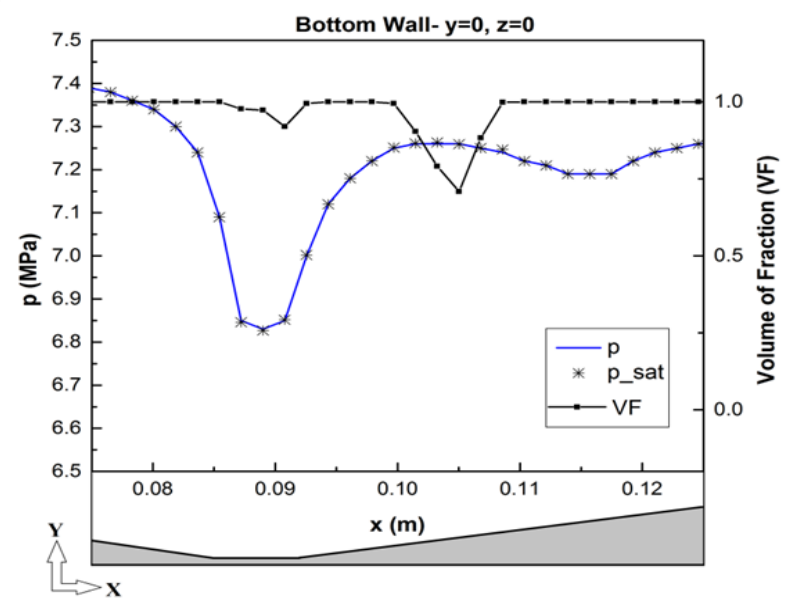

(c)

Figure 9. (a) Contours of $V F$ on the bottom wall at $t=2.0 \mathrm{~ms}$ for $\dot{m}=0.065 \mathrm{~kg} / \mathrm{s}$; average pressure, saturation pressure, and $V F$ vs. downstream direction for the bottom wall on (b) $z=-1.5 \mathrm{~mm}$, (c) $z=0$. 


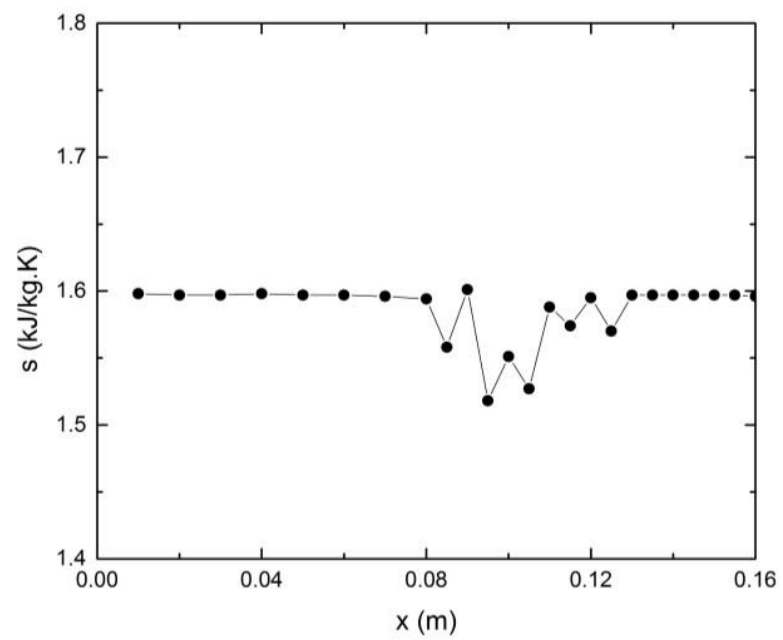

Figure 10. Entropy variation vs. downstream location for at $t=2.0 \mathrm{~ms}$ and $\dot{m}=0.065 \mathrm{~kg} / \mathrm{s}$ on the central plane $z=0$.

Figure 10 shows the entropy variation in the nozzle at $t=2 \mathrm{~ms}$ for $\dot{m}=0.065 \mathrm{~kg} / \mathrm{s}$ on the central plane. Entropy at each downstream location has been calculated as a function of the average temperature, average pressure, and average quality in the $y$-direction. As this graph shows, except for around the throat region and the divergent section of the nozzle, i.e. $0.08 \mathrm{~m}<\mathrm{x}<0.125 \mathrm{~m}$, associated with the location of the twophase mixture, the flow can be assumed isentropic.

As discussed earlier, Table 1 outlined the inlet temperature, pressure, and velocity magnitude for mass flow rates equal to $0.050,0.052$, and $0.065 \mathrm{~kg} / \mathrm{s}$. Note that the exit pressure is fixed at $2.28 \mathrm{MPa}$ for all cases. Thus, by increasing the mass flow rate, the inlet pressure and temperature increase and inspecting the results for different mass flow rates reveals the sensitivity of the nucleation process to the inlet conditions for the same nozzle geometry as is shown in Figures 11 and 12. Figure 11 (a) and (b) demonstrate the Mach number and volume fraction variations vs. $x$ for different mass flow rates with the fixed exit pressure. The speed of sound for the mixture has been calculated for homogenous frozen conditions, i.e., $\frac{1}{c^{2}}=\left(\rho_{l} V F_{l}+\rho_{g} V F_{g}\right) \times\left[\frac{V F_{l}}{\rho_{l} c_{l}^{2}}+\frac{V F_{g}}{\rho_{g} c_{g}^{2}}\right][19]$, where $c$ and $V F$ are the speed of sound and volume fraction and subscripts $l$ and $g$ refer to the liquid and gas phases, respectively. 


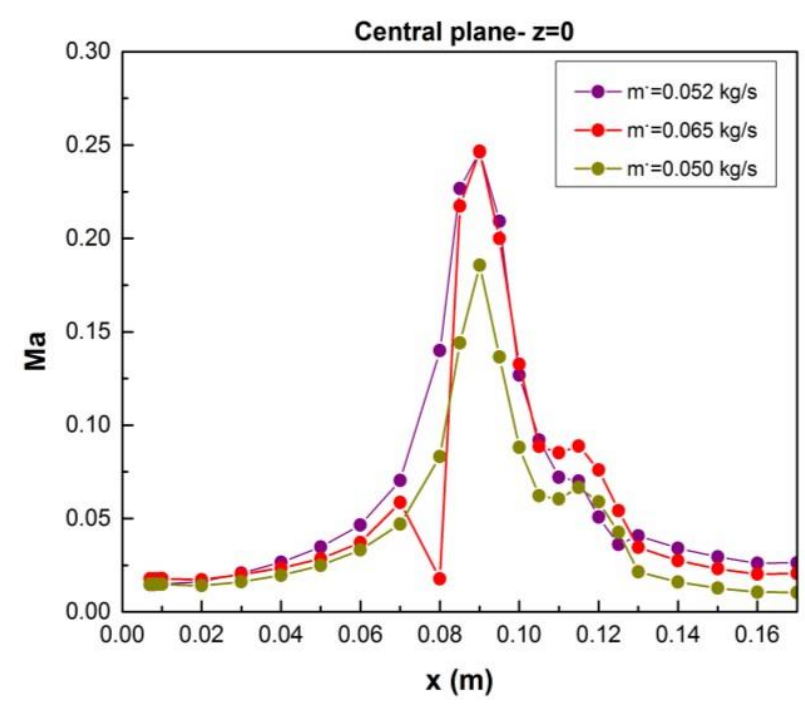

(a)

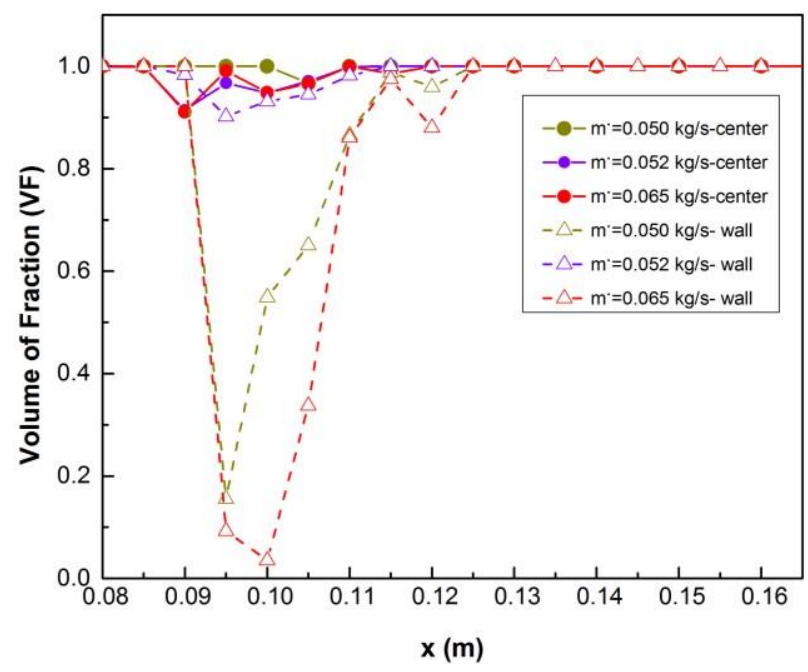

(b)

Figure 11. (a) Mach number vs. $x$ for different mass flow rates at the central plane, i.e. $z=0$ (b) $V F$ vs. $x$ for different mass flow rates for $z=0$ and $z=-1.5 \mathrm{~mm}$.

The homogenous frozen expression of speed of sound is based on the assumption of no mass-exchange between the two phases. As Figure 11 (a) shows, $M a$ increases to its maximum value near the throat and decreases downstream as was expected for subsonic flows. By increasing the mass flow rate, $M a$ increases due to the fixed exit pressure boundary conditions in these simulations that allows larger inlet pressure for higher mass flow rates and higher velocity within the throat area to reach to the same exit pressure for all mass flow rates. The drop in $M a$ for mass flow rate equal to $0.065 \mathrm{~kg} / \mathrm{s}$ near $x=0.08 \mathrm{~m}$ is due to larger content of liquid, i.e. smaller $V F$ as indicated in Figure 11 (b). $M a$ increases slightly downstream of the throat in the divergent section, i.e. $x=0.11 \mathrm{~m}$, since $V F$ increases (more vapor is formed) and the speed of sound decreases in vapor phase. As Figure 11 (b) indicates, $V F$ decreases drastically near the walls for all mass flow rates compared to that of the central plane. This is consistent with the conclusion obtained earlier that significant condensation occurs near the walls. $V F$ decreases by increasing the mass flow rate near the walls. Simulations with higher mass flow rates up to $0.095 \mathrm{~kg} / \mathrm{s}$ (not shown here), indicated that the flow 
remained subsonic throughout the nozzle since there was not significant vaporization downstream of the throat and throat region. The high liquid-content mixture causes a relatively low $M a$ in the two-phase regions as it increases the speed of sound and decreases $M a$.

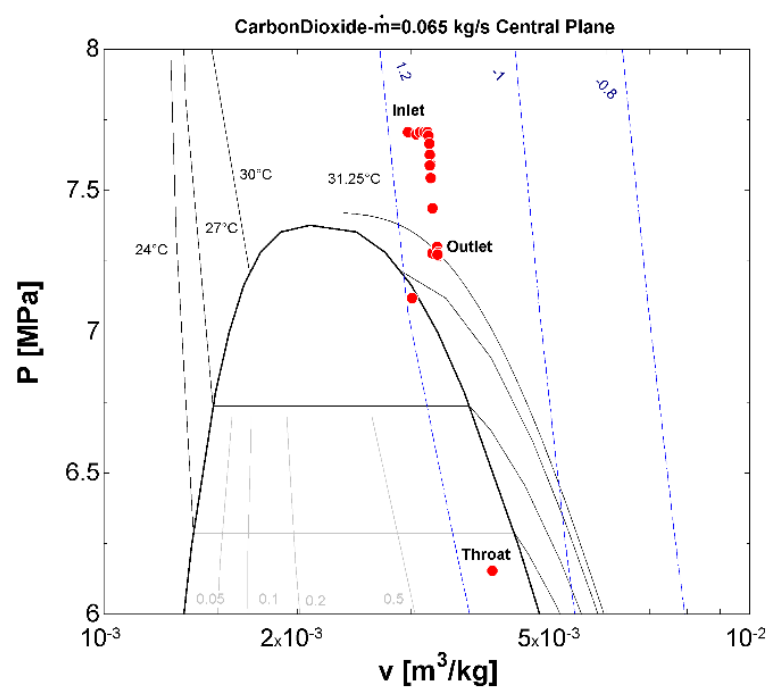

(a)

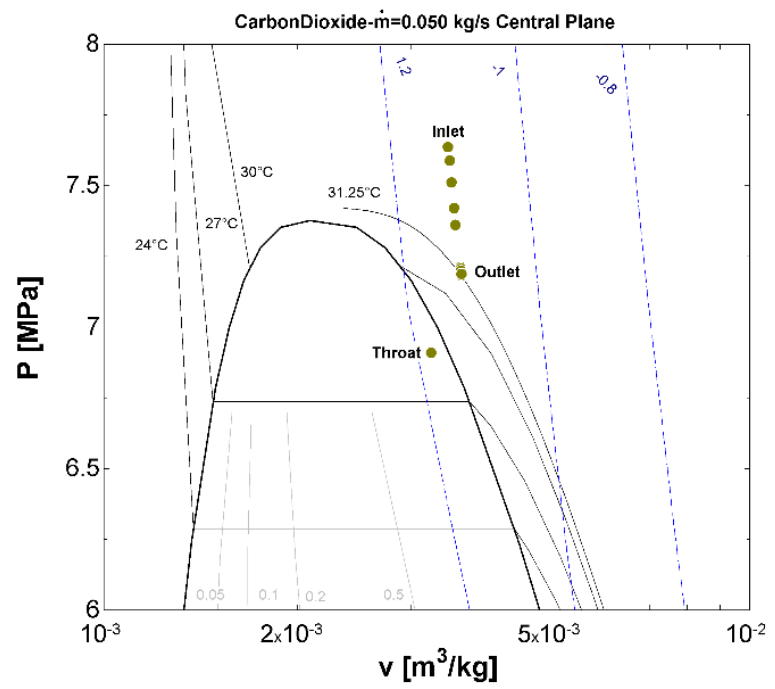

(c)

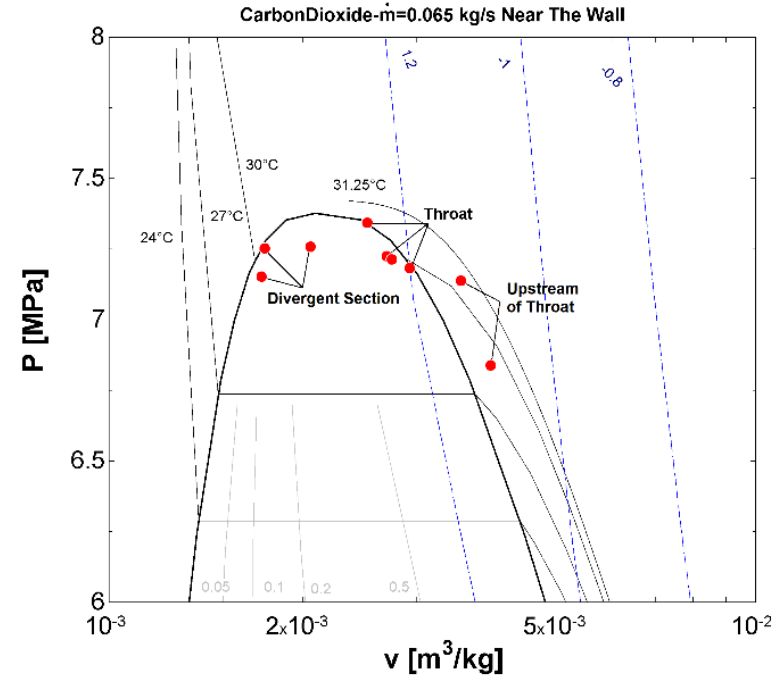

(b)

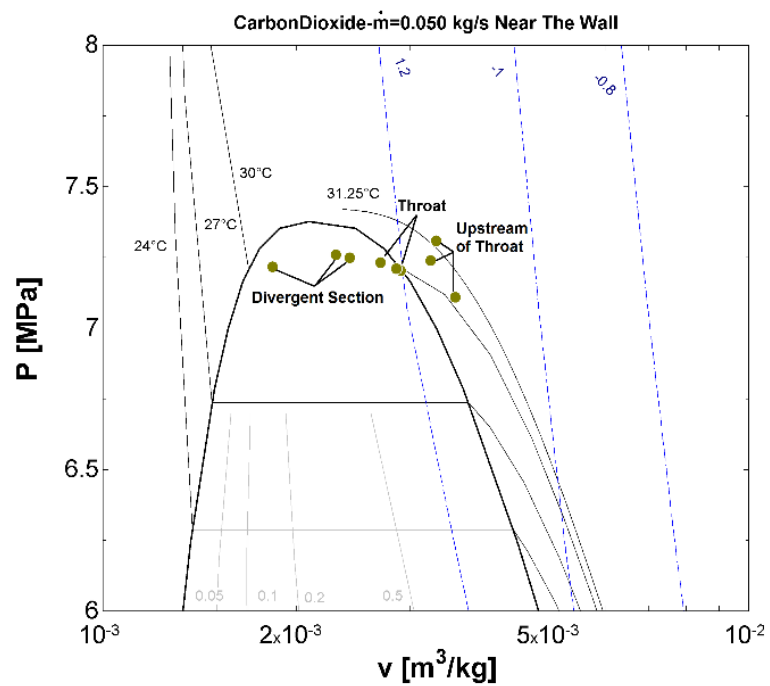

(d)

Figure 12. $P-v$ diagrams for (a) $\dot{m}=0.065 \mathrm{~kg} / \mathrm{s}$ on the central plane $(z=0)$; (b) $\dot{m}=0.065 \mathrm{~kg} / \mathrm{s}$ near the wall $(z=-1.5 \mathrm{~mm}$.); (c) $\dot{m}=0.050 \mathrm{~kg} / \mathrm{s}$ on the central plane; (d) $\dot{m}=0.050 \mathrm{~kg} / \mathrm{s}$ near the wall. 
Figure 12 shows the state of $\mathrm{CO}_{2}$ within the nozzle with respect to the vapor dome in $P$-v diagram for mass flow rates equal to $0.065 \mathrm{~kg} / \mathrm{s}$ and $0.050 \mathrm{~kg} / \mathrm{s}$ for the central plane ( $a$ and $c$ ) and near the wall $(b$ and d), respectively. Each point on these plots represent different locations within the nozzle, i.e. inlet, throat, divergent section, and outlet at $t=2.0 \mathrm{~ms}$. As Figure 12 (b) and (d) indicate, the pressure drop from the near-critical conditions to sub-critical is smaller near the wall for both mass flow rates. However, the mixture changes from saturated liquid to saturated vapor. As observed in Figure 11 (b), $V F$ is smaller for larger mass flow rates within the two-phase region. On the contrary, the pressure drop is larger at the center of the nozzle since the velocity drop is larger for both mass flow rates as indicated in Figure 12 (b) and (d). For these conditions, $V F$ remains close to one, i.e. more vapor than liquid exists compared to that of the near walls. The higher pressure drop observed for higher mass flow rates leads to lower $V F$ and quality on the central plane as the comparison between Figure 12 (a) and (c) shows. Therefore, it can be emphasized again that cavitation effects are not as significant as condensation near the walls for the near-critical inlet conditions.

The simulation results for different mass flow rates, i.e. pressure, quality, and temperature have been averaged along the nozzle height ( $y$-direction) at each axial location $x$, and the averaged values have been used to calculate the nucleation rate at each axial location for the central plane and near the walls of the nozzle. It is noted that since homogenous equilibrium model has been assumed in our simulations, at each cell, equilibrium between the two phases is expected; thus, the nucleation rate is zero before averaging the results. However, to compare the nucleation behavior for different mass flow rates and at different axial locations within the nozzle, the averaged values have been used. Therefore, for the conditions where the average pressure is smaller or larger than the saturation pressure and $V F$ at that average temperature is very close to one, the nucleation rate is not zero. Obtaining nucleation rate at different locations within the nozzle will be helpful to study the nucleation process as is shown below.

Nucleation of a new phase form the non-equilibrium conditions depends on the nucleation work, i.e. the Gibbs free energy barrier [20]. A relative time scale ratio $t_{c r}=t_{r} / t_{n}$ can be defined based on the classical nucleation theory, where $t_{r}=l / u_{\text {avg }}$ is the residence time and $l$ and $u_{\text {avg }}$ are the length of the condensing volume 
and the average flow velocity, respectively [12]. $t_{n}=\left(J_{\max } \mathrm{V}\right)^{-1}$ is the nucleation time and $J_{\max }$ is the maximum nucleation rate obtained from Eq. 7. The critical radius of the condensed droplets and limiting energy barrier for nucleation can be obtained from Eq. 8 and 9 and the Gibbs free energy can be calculated from Eq. 10.

Nucleation Rate

$$
J_{\max }=\left[\sqrt{\frac{2 \sigma}{\pi m^{3}} \frac{\rho_{v}^{2}}{\rho_{l}}}\right] e^{\left(-\frac{\Delta G^{*}}{\kappa T}\right)}
$$

Critical Radius

$$
r^{*}=\frac{2 \sigma}{\rho_{l}\left[g\left(p_{v}, T\right)-g\left(p_{s}, T\right)\right]}
$$

Limiting Energy Barrier $\Delta G^{*}=\frac{4}{3} \pi r^{* 2} \sigma$

Gibbs Free Energy $\quad g\left(p_{v}, T\right)-g\left(p_{s}, T\right)=\left[h\left(p_{v}, T\right)-T s\left(p_{v}, T\right)\right]-\left[h\left(p_{s}, T\right)-T s\left(p_{s}, T\right)\right]$;

where $\sigma, \rho_{v}, \rho_{l}, m, \kappa, G, T, p_{s}$, and $r^{*}$ are surface tension coefficient, gas-phase density, liquid-phase density, molecular weight, Boltzmann's constant, Gibbs free energy, temperature, saturation pressure at the given temperature, and critical radius, respectively. The time scale determines whether the deviation from the equilibrium conditions in an internal flow is large enough for stable droplets to form. Ratios below one indicate that the nucleation time is larger than the residence time, implying that condensation cannot occur. For our base calculation, the obtained resident and nucleation time were $t_{r}=\frac{l}{u_{\text {avg }}} \approx 10^{-5} s$ and $t_{n} \approx 10^{-30} s<<t_{r}$ showing that very close to the critical point nucleation occurs. The condensation length in these calculations is associated with the location where $V F<1$ in the simulations results.

Figure 13 shows the nucleation rate as a function of $x$ for mass flow rates equal to $0.050,0.052$, and $0.065 \mathrm{~kg} / \mathrm{s}$ for central plane and near the wall, i.e. $z=0$ and $z=-1.5 \mathrm{~mm}$, respectively. In the throat area $(0.085 \mathrm{~m}<x<0.095 \mathrm{~m})$, nucleation on the central plane is larger than the wall for mass flow rates equal to 0.065 and $0.052 \mathrm{~kg} / \mathrm{s}$, and larger for $0.065 \mathrm{~kg} / \mathrm{s}$. This is consistent with the contours of $V F$ for the central plane shown in Figure 4. In the divergent section, i.e. $x \geq 0.10 \mathrm{~m}$, nucleation on the wall is larger than that of the center for all cases. Further downstream, i.e. $0.10 \mathrm{~m}<x \leq 0.12 \mathrm{~m}$ for $0.065 \mathrm{~kg} / \mathrm{s}$, the average velocity 
is higher; therefore, the pressure drop is sufficient to initiate the phase change. For mass flow rate equal to $0.050 \mathrm{~kg} / \mathrm{s}$, nucleation occurs downstream in the throat area, i.e. $x=0.095 \mathrm{~m}$ compared to larger mass flow rates, i.e. $m^{\circ}=0.052$ and $0.065 \mathrm{~kg} / \mathrm{s}$ since the lower pressure drop in the throat area prevents nucleation upstream of the throat at $x=0.085 \mathrm{~m}$. The pressure drop required for phase change is obtained at $x=0.095$ $\mathrm{m}$ for this lower mass flow rate. However, more condensation occurs near the wall than cavitation due to the lower velocity and higher pressure near the wall, leading to higher nucleation rates compared to the central plane throughout the nozzle for mass flow rate equal to $0.050 \mathrm{~kg} / \mathrm{s}$.

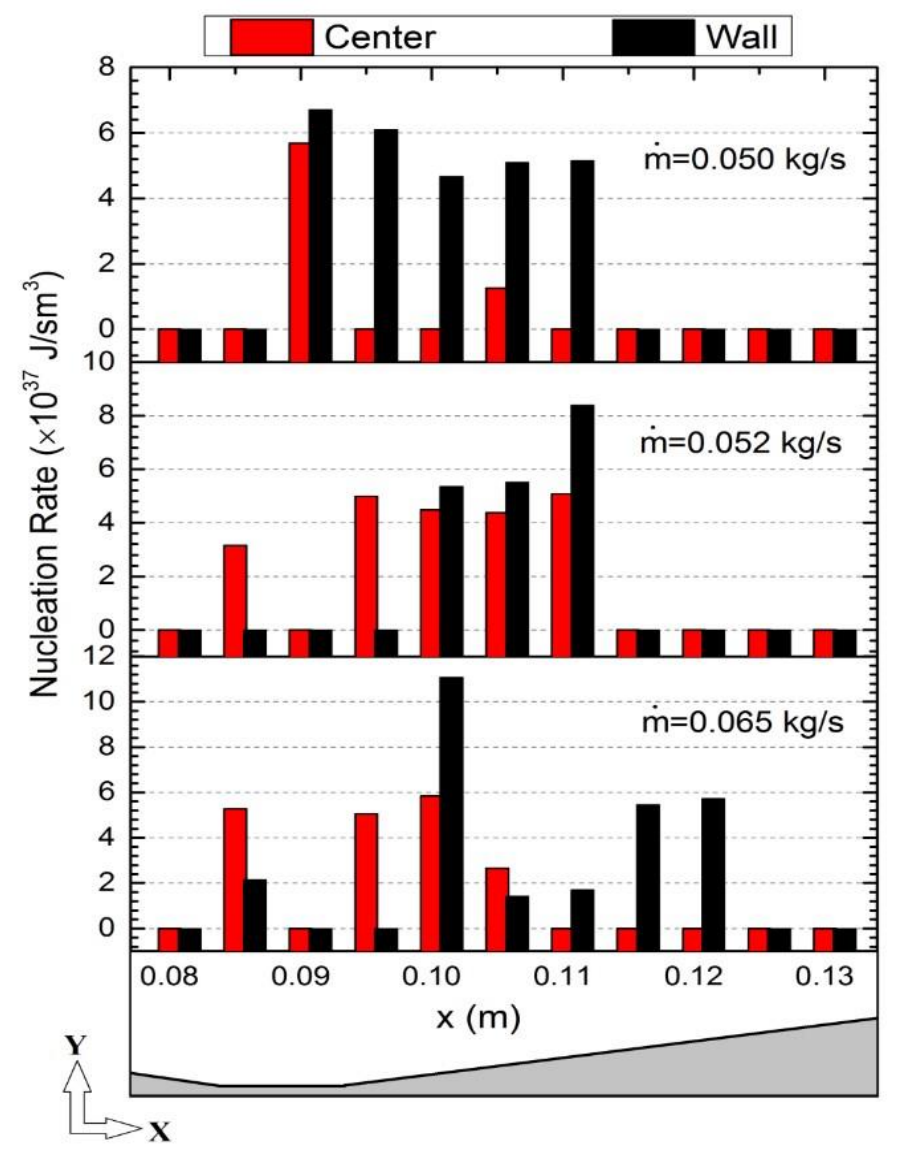

Figure 13. Nucleation rate vs. $x$ for central plane and wall for $\dot{m}=0.050,0.052$, and $0.065 \mathrm{~kg} / \mathrm{s}$.

Previous studies of radial compressor operating with supercritical $\mathrm{CO}_{2}$ at realistic cycle conditions showed that away from the critical point, i.e. inlet pressure equal to $7.69 \mathrm{MPa}$, condensation occurred at 
very small regions near the blade tip and blade trailing edge [8]. Their studies showed that for higher rotational speeds (leading to higher pressure drop at the compressor inlet) and higher mass flow rates the regions in which condensation was predicted were considerably larger. The presented computational conditions are of relevance to the real compressor inlet conditions as stated above; indicating that even a small pressure drop near the critical point leads to a large volume of liquid (with respect to the size of the nozzle) downstream of the throat that moves downstream of the nozzle and cavitation in the sharp corners of the throat. Thus, we predict that condensation and cavitation at conditions near the critical point affect compressor performance; especially, at higher mass flow rates, the nucleation is more likely to occur due to larger pressure drops. Further experimental data are required to demonstrate the effects of nucleation on compressor performance near the critical point.

\section{Summary \& Conclusions}

Nucleation of $\mathrm{S}-\mathrm{CO}_{2}$ very close to the critical pressure and temperature flowing through a convergingdiverging nozzle was computationally studied. 3D transient compressible Navier-Stokes and energy equations were solved in OpenFOAM. Developed fluid property interpolation tables (FIT) based on a piecewise biquintic spline interpolation of Helmholtz energy was integrated with OpenFOAM to model S$\mathrm{CO}_{2}$ properties. The mass fraction of vapor created in the venturi was calculated using homogeneous equilibrium model (HEM). The proposed nozzle geometry provided the required pressure drop to develop two-phase flow by keeping the inlet at supercritical pressure and temperature in the vicinity of the critical point. $1 D$ isentropic model of the nozzle indicated that nucleation process was very sensitive to the initial conditions. The 3D simulations demonstrated that the nucleation first occurred in the throat area in the center of the channel. However, the nucleation took place in the divergent section near the wall and then convected downstream later in time. Nucleation in central plane was associated with larger pressure drop and higher vapor-content (higher $V F$ ); whereas lower pressure drop and more liquid-content (lower $V F$ ) was observed near the walls. The two-phase mixture was convected downstream and pushed away from the walls due to re-circulation region near the top wall. Nucleation rate, $M a$, and $V F$ increased with increasing 
the mass flow rate at constant exit pressure. For the smallest mass flow rate tested, i.e. $m^{\circ}=0.050 \mathrm{~kg} / \mathrm{s}$, the pressure drop required for nucleation was obtained downstream of the throat and the nucleation rate was larger near the wall everywhere. It was observed that condensation, i.e. formation of stable liquid droplets near the walls due to deceleration were more significant than cavitation in near-critical conditions.

The presented computational conditions are of relevance to the real compressor inlet conditions; indicating that even a small pressure drop near the critical point leads to a large volume of liquid (with respect to the size of the nozzle) downstream of the throat that moves downstream of the nozzle and cavitation in the sharp corners of the throat. Thus, we predict that condensation and cavitation near the critical point might affect compressor performance; especially, at higher mass flow rates, the nucleation is more likely to occur due to larger pressure drops.

\section{Acknowledgment}

Support for this research was provided by the U.S. Department of Energy, Nuclear Energy University Programs (NEUP) via project number NEUP 14-6670.

\section{References}

1. Angelino, G., 1969, “Carbon dioxide condensation cycles for power production”, Journal of Engineering for Power-T. ASME, 10, pp. 272-287.

2. Feher, E. G., 1968, “The supercritical thermodynamic power cycle”, Energy Conversion, 8, pp. 8590.

3. Dostal, V., Hejzlar, P., and Driscoll, M. J., 2006, "High performance supercritical carbon dioxide cycle for next generation nuclear reactors", Nuclear Technology, 154(3), pp. 265-282.

4. Dostal, V., Hejzlar, P., and Driscoll, M. J., 2006, "The supercritical carbon dioxide power cycle: Comparison to other advanced power cycles", Nuclear Technology, 154(3), pp. 283-301.

5. Ma, Z., Turchi, C. "Advanced supercritical carbon dioxide power cycle configurations for use in concentrating solar power systems", Proceedings of Supercritical $\mathrm{CO}_{2}$ Power Cycle Symposium 
2011 May 24, pp. 24-25.

6. Persichilli, M., Kacludis, A., Zdankiewicz, E. and Held, T., 2012, "Supercritical CO2 Power Cycle Developments and Commercialization: Why sCO2 can Displace Steam”, Power-Gen India \& Central Asia 2012, 19-21 April, 2012, Pragati Maidan, New Delhi, India.

7. Muto, Y., Ishiyama, S., Kato, Y., Ishizuka, T., \& Aritomi, M., 2010, “Application of Supercritical CO2 Gas Turbine for the Fossil Fired Thermal Plant”, Journal of Energy and Power Engineering, 4(9), pp.7-19.

8. Pecnik, R., Rinaldi, E., and Colonna, P., 2012, "Computational fluid dynamics of a radial compressor operating with supercritical $\mathrm{CO}_{2}$ ”, Journal of Engineering for Gas Turbines and Power, 134(12), pp. 1223011-1223018.

9. Takagi, K., Muto, Y., Ishizuka, T., Kikura, H., and Aritomi, M., 2010, "Research on flow characteristics of supercritical $\mathrm{CO}_{2}$ axial compressor blades by CFD analysis", Journal of Power and Energy Systems, 4(1), pp. 138-149.

10. Nakagawa, M., Berana, M. S., and Kishine, A., 2009, "Supersonic two-phase flow of $\mathrm{CO}_{2}$ through converging-diverging nozzles for the ejector refrigeration cycles", International Journal of Refrigeration, 32(6), pp. 1195-1202.

11. Yazdani, M., Alahyari, A., and Radcliff, T., 2014, "Numerical modeling and validation of supersonic two-phase flow of $\mathrm{CO}_{2}$ in converging-diverging nozzles", Journal of Fluid Engineering, 136, pp. 0145031-6.

12. Lettieri, C, Yang, D., and Spakovszky, Z., 2015, “An investigation of condensation effects in supercritical carbon dioxide compressors", Journal of Engineering for Gas Turbines and Power, 137, pp. 0826021-8.

13. Baltadjiev ND, Lettieri C, and Spakovszky Z., 2015, “An investigation of real gas effects in supercritical $\mathrm{CO}_{2}$ centrifugal compressors”, ASME Journal of Turbomachinery, 137(9), pp. 0910031-13.

14. OpenFOAM, 2012, "User Guide 2.1.1". [Online] 
15. Yuan, H., Pidaparti, Wolf, M., Edlebeck, J., Anderson, M., 2015, "Numerical modeling of supercritical carbon dioxide flow in see-through labyrinth seals", Nuclear Engineering and Design, 293, pp. 436-446.

16. Ghiaasiaan, S. Mostafa, 2007, "Two-phase flow, boiling, and condensation: in conventional and miniature systems", Cambridge University Press.

17. Zivi, S. M. 1964. "Estimation of steady-state steam void-fraction by means of the principle of minimum entropy production", Transactions ASME, Journal of Heat Transfer, Series C, 86, pp. 247-252.

18. Smith, S. L. 1969, "Void fractions in two-phase flow: a correlation based upon an equal velocity head model”, Proceedings of the Institution of Mechanical Engineers, 184(1), pp. 647-664.

19. Brennen, C. E., 2005, “Fundamentals of multiphase flow”, Cambridge University Press, New York.

20. McDonald, J. E., 1962, “Homogeneous nucleation of vapour condensation. I. thermodynamic aspects," American Journal of Physics, 30(12), pp. 870-877. 